IPMU-11-0191

CALT-68-2855

MPP-2011-130

\title{
The ABCDEFG of Instantons and W-algebras
}

\author{
Christoph A. Keller ${ }^{\sharp}$, Noppadol Mekareeya ${ }^{b}$, Jaewon Song ${ }^{\sharp}$, and Yuji Tachikawa ${ }^{\natural}$ \\ \# California Institute of Technology, Pasadena, CA 91125, USA \\ b Max-Planck-Institut für Physik (Werner-Heisenberg-Institut), \\ Föhringer Ring 6, 80805 München, Deutschland \\ घ IPMU, University of Tokyo, Kashiwa, Chiba 277-8583, Japan
}

\begin{abstract}
For arbitrary gauge groups, we check at the one-instanton level that the Nekrasov partition function of pure $\mathcal{N}=2$ super Yang-Mills is equal to the norm of a certain coherent state of the corresponding $\mathrm{W}$-algebra. For non-simply-laced gauge groups, we confirm in particular that the coherent state is in the twisted sector of a simply-laced W-algebra.
\end{abstract}




\section{Contents}

1 Introduction $\quad 1$

2 Instanton calculation $\quad 5$

2.1 Generalities ....................... 5

2.2 One-instanton contribution . . . . . . . . . . . . . 6

3 Free field realization of W-algebras

3.1 Simply laced $\mathrm{W}$-algebras . . . . . . . . . . . . . . . 8

3.2 Twisted sectors of the simply-laced W-algebras . . . . . . . . . . . . . 9

3.3 Basic properties of the Verma module . . . . . . . . . . . . 10

4 Instantons and coherent states of W-algebras 11

4.1 Identification of the coherent state . . . . . . . . . . . . . . 11

4.2 Coherent state at the lowest level . . . . . . . . . . . . . . . 12

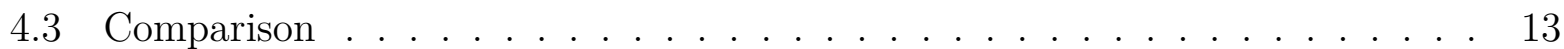

A Roots of simple Lie algebras $\quad 18$

A.1 Simply-laced algebras . . . . . . . . . . . . . . . . 18

A.2 Non-simply-laced algebras . . . . . . . . . . . . . . . . . . . . 19

B Hilbert series of the one-instanton moduli space 21

C Kac determinant at the lowest level

D Construction of the W-algebra of type $E_{6} \quad 25$

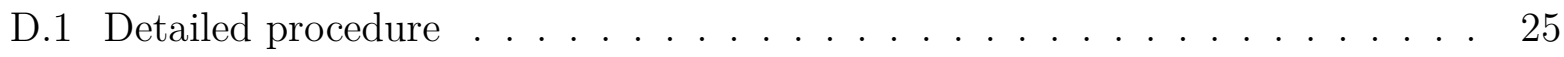

D.2 Explicit generators . . . . . . . . . . . . . . . . . 26

\section{Introduction}

It has been almost twenty years since it was realized that the quantum dynamics of $4 \mathrm{~d}$ $\mathcal{N}=2$ gauge theory is encoded in the classical geometry of a two-dimensional Riemann surface $\Sigma$, called the Seiberg-Witten curve [1, 2]. The curve $\Sigma$ was originally introduced as an auxiliary construct, but it was later recognized [3, 4, 5, 6] that $\Sigma$ is a branched covering of another two-dimensional surface $C$ on which a $6 \mathrm{~d} \mathcal{N}=(2,0)$ theory is compactified to obtain the $4 \mathrm{~d} \mathcal{N}=2$ theory.

From this point of view, it is not surprising that the $2 \mathrm{~d}$ quantum dynamics on $\Sigma$ or $C$ have some bearing on the $4 \mathrm{~d}$ gauge dynamics. Indeed the role of $2 \mathrm{~d}$ free bosons in this setup has long been recognized: see e.g. [7, 8, 9] for the identification of the gravitational factor 


\begin{tabular}{l||r|l||r||r|l|l}
$G$ & $h^{\vee}$ & $w_{i}$ & $G$ & $h^{\vee}$ & $w_{i}$ & $\Gamma^{(r)}$ \\
\hline$A_{n-1}$ & $n$ & $2,3, \ldots, n$ & $B_{n}$ & $2 n-1$ & $2,4, \ldots, 2 n$ & $A_{2 n-1}^{(2)}$ \\
$D_{n}$ & $2 n-2$ & $2,4, \ldots, 2 n-2 ; n$ & $C_{n}$ & $n+1$ & $2,4, \ldots, 2 n$ & $D_{n+1}^{(2)}$ \\
$E_{6}$ & 12 & $2,5,6,8,9,12$ & $F_{4}$ & 9 & $2,6,8,12$ & $E_{6}^{(2)}$ \\
$E_{7}$ & 18 & $2,6,8,10,12,14,18$ & $G_{2}$ & 4 & 2,6 & $D_{4}^{(3)}$ \\
$E_{8}$ & 30 & $2,8,12,14,18,20,24,30$ & & & &
\end{tabular}

Table 1: The dual Coxeter number $h^{\vee}$ and the dimensions $w_{i}$ of the Casimir invariants for all simple Lie groups $G$. Recall $A_{n-1}=\mathrm{SU}(n), B_{n}=\mathrm{SO}(2 n+1), C_{n}=\mathrm{Sp}(n), D_{n}=\mathrm{SO}(2 n)$. For non-simply-laced $G$, the Langlands dual $\left(G^{(1)}\right)^{\vee}=\Gamma^{(r)}$ of the affine $G$ algebra is also shown.

on the $u$-plane [10, 11] as the one-loop determinant of a free boson on $\Sigma$, and [12, 13, 14] for a more general analysis. More recently, it was noticed that $2 \mathrm{~d}$ Toda field theories with W-symmetry arise naturally on $C$ by considering the partition function on $S^{4}$ of the gauge theory [15, 16]. This correspondence has been studied thoroughly for gauge groups $\mathrm{SU}(N)^{1}$ but not much work has been done for gauge groups of other types, see e.g. [17, 18, 19, 20].

The aim of this paper is to rectify the situation, by studying $\mathcal{N}=2$ pure gauge theory for arbitrary $G$ as an extension of [21, 22, 23]. The main tools are the Hilbert series of the moduli space for arbitrary gauge group $G$ found and discussed e.g. in [24, 25, 26], the free-field realization of W-algebras [27, 28, 29], and K. Thielemans' Mathematica package OPEdefs.m [30].

Martinec-Warner solution of $\mathcal{N}=2$ pure theory: The Seiberg-Witten curve of pure $\mathcal{N}=2$ gauge theory for arbitrary $G$ was constructed in [31] as the spectral curve of a Toda lattice. (See also [32, 33, 34, 35].) From a modern perspective [3, 5, 6], their construction reads as follows. Let us first consider the case when $G$ is simply-laced. Take $6 \mathrm{~d} \mathcal{N}=(2,0)$ theory of type $G$, and compactify this theory on $C=\mathbb{C P}^{1}$ parametrized by $z$, with two codimension 2 defects at $z=0$ and $z=\infty$. The 6 d theory has world-volume fields $\phi^{\left(w_{i}\right)}(z)$ on $C$, transforming as degree $w_{i}$ multi-differentials, where $w_{i}$ is the degree of the Casimir invariants of $G$ given in Table 1. We then set

$$
\begin{array}{ll}
\phi^{\left(w_{i}\right)}(z)=u^{\left(w_{i}\right)}(d z / z)^{w_{i}}, & \left(w_{i} \neq h^{\vee}\right) ; \\
\phi^{\left(h^{\vee}\right)}(z)=\left(\Lambda^{h^{\vee}} z+u^{\left(h^{\vee}\right)}+\frac{\Lambda^{h^{\vee}}}{z}\right)(d z / z)^{h^{\vee}} & \left(w_{i}=h^{\vee}\right) .
\end{array}
$$

Here $u^{\left(w_{i}\right)}$ is the vev of the dimension $w_{i}$ Coulomb branch operator, and $\Lambda$ is the holomorphic dynamical scale of the gauge theory. From this data one can then construct the Seiberg-

\footnotetext{
${ }^{1}$ The authors apologize for not citing papers on $\mathrm{SU}(N)$.
} 


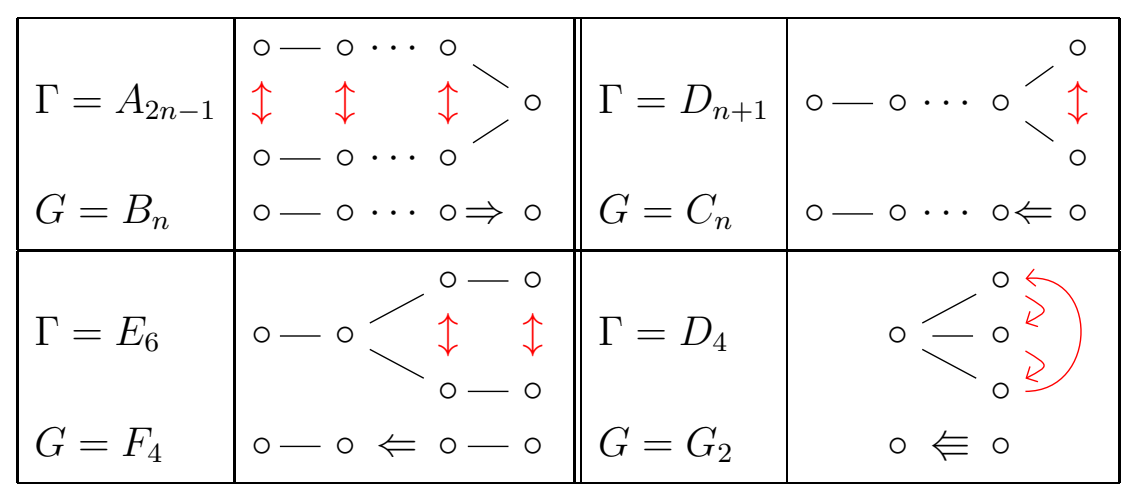

Figure 1: The relation between a non-simply-laced Lie algebra $G$, its associated simply-laced algebra $\Gamma$, and the outer automorphism used to fold $\Gamma$ to obtain $G$.

Witten curve $\Sigma$ [36], or equivalently the fibration of the ALE space of type $G$ [35, 37]. In the following we label the degrees $w_{i}$ so that $h^{\vee}=w_{n}$.

When $G$ is non simply-laced, we take a pair $\left(\Gamma, \mathbb{Z}_{r}\right)$ such that the twisted affine Lie algebra $\Gamma^{(r)}$ is the Langlands dual to $G^{(1)}$, the untwisted affine algebra of $G$. In other words, the Dynkin diagram of $G$ is obtained by folding the Dynkin diagram of $\Gamma$ as in Fig. $1^{2}$ For example, when $G=G_{2}, \Gamma=\mathrm{SO}(8)$ and $r=3$. We then put $6 \mathrm{~d}$ theory of type $\Gamma$ on $C=\mathbb{C P}^{1}$, with a twist by $\mathbb{Z}_{r}$ around two singularities at $z=0, \infty$. The fields of the $6 \mathrm{~d}$ theory are divided into two sets, $\phi^{\left(\hat{w}_{i}\right)}(z)$ which are invariant under $\mathbb{Z}_{r}$ action, and $\phi^{\left(\tilde{w}_{i}\right)}(z)$ which transform nontrivially under $\mathbb{Z}_{r}$. We then take

$$
\begin{array}{ll}
\phi^{\left(\hat{w}_{i}\right)}(z)=u^{\left(\hat{w}_{i}\right)}(d z / z)^{\hat{w}_{i}}, & \\
\phi^{\left(\tilde{w}_{i}\right)}(z)=0, & \left(\tilde{w}_{i} \neq h^{\vee}\right), \\
\phi^{\left(\tilde{w}_{i}\right)}(z)=\left(\Lambda^{\tilde{w}_{i}} z^{1 / r}+\frac{\Lambda^{\tilde{w}_{i}}}{z^{1 / r}}\right)(d z / z)^{\tilde{w}_{i}} & \left(\tilde{w}_{i}=h^{\vee}\right) .
\end{array}
$$

Here, $\Lambda$ is the dynamical scale and $u^{\left(\hat{w}_{i}\right)}$ is the vev of the degree- $\hat{w}_{i}$ Coulomb branch operator; note that the degrees of Casimirs of $G$ are exactly the degrees of Casimirs of $\Gamma$ invariant under $\mathbb{Z}_{r}$. Note also that the dual Coxeter number of $G$ are exactly the highest degree of Casimirs of $\Gamma$ not invariant under $\mathbb{Z}_{r}$.

The construction is summarized in Fig. 2; the $6 \mathrm{~d} \mathcal{N}=(2,0)$ theory of type $\Gamma$ on a circle with $\mathbb{Z}_{r}$ twist gives maximally supersymmetric 5d Yang-Mills theory of gauge group $G$. To obtain pure $\mathcal{N}=2$ Yang-Mills theory, we need to put the 5 d Yang-Mills theory on a segment with an appropriate half-BPS boundary condition on both ends. The boundary condition then becomes the prescribed singularity of the worldvolume fields $\phi^{\left(w_{i}\right)}(z)$. When $G$ is classical, the 5d Yang-Mills theory can be realized on coincident D4-branes, possibly

\footnotetext{
${ }^{2} \Gamma$ is called the associated simply-laced algebra of $G[38 . G$ is also known as the orbit Lie algebra of the pair $\left(\Gamma, \mathbb{Z}_{r}\right)$, see [39]. Note that $G$ is not the $\mathbb{Z}_{r}$-invariant part of $\Gamma$ in general, as explained in Appendix A.2.
} 


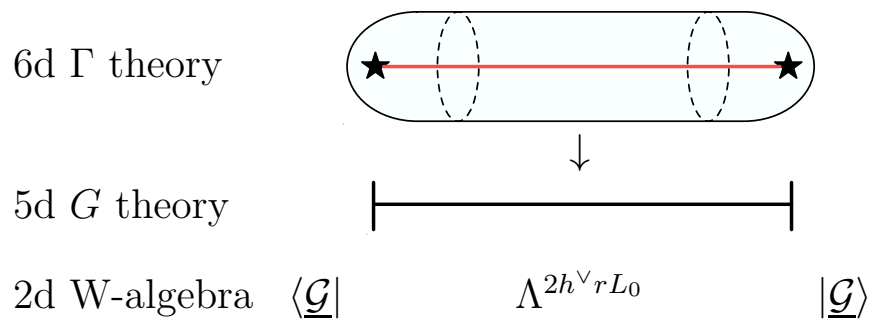

Figure 2: Top: the Seiberg-Witten solution of pure $\mathcal{N}=2$ super Yang-Mills theory with gauge group $G$ in terms of $6 \mathrm{~d} \mathcal{N}=(2,0)$ theory of type $\Gamma$ on $C=\mathbb{C P}^{1}$ with the $\mathbb{Z}_{r}$ twist line from $z=0$ to $z=\infty$. Middle: the $S^{1}$ reduction to the 5 d maximally supersymmetric Yang-Mills theory with gauge group $G$ on a segment, with a suitable half-BPS boundary condition on both ends. Bottom: In the $2 \mathrm{~d}$ description, the coherent state $\langle\underline{\mathcal{G}}|$ is produced by the BPS boundary condition. It is then propagated along the horizontal direction and annihilated by $|\underline{\mathcal{G}}\rangle$.

on top of an O4-plane. Then the BPS boundary condition comes from ending them on an NS5-brane [4, 40, 41, 42].

Correspondence with $\mathrm{W}$-algebra: The essence of the $2 \mathrm{~d}-4 \mathrm{~d}$ correspondence is that the correlator of the $2 \mathrm{~d}$ Toda theory equals the Nekrasov partition function of the $4 \mathrm{~d}$ theory with $\Omega$ deformation parameters $\epsilon_{1,2}$, and that the vevs of its $\mathrm{W}$-currents $W^{\left(w_{i}\right)}(z)$ become the world-volume fields $\phi^{\left(w_{i}\right)}$ determining the Seiberg-Witten curve:

$$
\lim _{\epsilon_{1,2} \rightarrow 0}\left\langle W^{\left(w_{i}\right)}(z)\right\rangle(d z)^{w_{i}} \rightarrow \phi^{\left(w_{i}\right)}(z) .
$$

In particular, the instanton contribution to Nekrasov's partition function should equal the conformal block of the $\mathrm{W}$-algebra. With the help of (1.6) we translate the conditions on the singularities of $\phi^{\left(w_{i}\right)}(z)$ at $z=0, \infty$ into conditions on the state $|\mathcal{G}\rangle$, which we call the Gaiotto-Whittaker state, inserted at $z=0, \infty$. This state turns out to be a certain coherent state in the Verma module of the untwisted sector of the $W(G)$-algebra when $G$ is simply-laced, and in the $\mathbb{Z}_{r}$-twisted sector of the $W(\Gamma)$-algebra when $G$ is non-simply-laced. We come back to the details in Sec. 4.1. The most important relation is

$$
W_{1 / r}^{\left(h^{\vee}\right)}|\mathcal{G}\rangle=\Lambda^{h^{\vee}}|\mathcal{G}\rangle .
$$

Under a suitable identification of parameters we should then have the equality

$$
Z_{\text {inst }}\left(\boldsymbol{a} ; \epsilon_{1,2}\right)=\langle\mathcal{G} \mid \mathcal{G}\rangle=\left\langle\underline{\mathcal{G}}\left|\Lambda^{2 h^{\vee} r L_{0}}\right| \underline{\mathcal{G}}\right\rangle .
$$

On the right hand side, $|\mathcal{G}\rangle$ is the coherent state (1.7) defined by setting $\Lambda=1$. The relation can be understood as in Fig. 2 , the boundary condition creates the state $\langle\mathcal{G}|$, which is then 
propagated by the distance $\propto \log \Lambda$, then is annihilated by $|\underline{\mathcal{G}}\rangle$. Indeed, $\log \Lambda$ is the UV coupling, and is proportional to the length of the fifth direction.

This relation was first considered for $\mathrm{SU}(2)$ in [21], and its calculation was later streamlined in [22]. The check for SU(3) was performed in [23] using the known explicit commutation relation of the $W_{3}$-algebra. In this paper we will check (1.8) at the one-instanton level uniformly for all $G$. We will use the free-field realization of the W-algebra, without explicitly writing down the complicated commutation relation of the modes of its modes. Before proceeding, it is to be noted that relation (1.8) when we have a full surface operator has already been rigorously proved for all groups to all order in [43, 44].

The remainder of this paper is organized as follows. In Sec. 2 we review how we obtain the instanton contribution to Nekrasov's partition function, and evaluate the one-instanton term for all $G$. In Sec. 3, we review the construction of the W-algebras in terms of free bosons. In Sec. 4, we identify the coherent state $|\mathcal{G}\rangle$ from the behavior of the worldvolume fields $\phi^{\left(w_{i}\right)}(z)$, and compare its norm to the instanton contribution to Nekrasov's partition function. Most of the calculations are relegated to the appendices.

\section{Instanton calculation}

\subsection{Generalities}

Nekrasov's partition function was introduced in [45], as a culmination of a long series of works e.g. [46, 47, 48] on the instanton calculation of the nonperturbative effects in $\mathcal{N}=2$ gauge theory. We follow the presentation of [49] in the following. Consider the partition function of the $5 \mathrm{~d}$ supersymmetric field theory with the same matter content on the spacetime of the form $\mathbb{C}^{2} \times \mathbb{R}$ parameterized by $\left(z_{1}, z_{2}, x_{5}\right)$, with the identification

$$
\left(z_{1}, z_{2}, x_{5}\right) \sim\left(z_{1} e^{\epsilon_{1} \beta}, z_{2} e^{\epsilon_{2} \beta}, x_{5}+\beta\right)
$$

together with an appropriate $\mathrm{SU}(2)_{R}$ symmetry rotation to preserve supersymmetry. The vev of the $4 \mathrm{~d}$ scalar field can be included either via the vev of the $5 \mathrm{~d}$ scalar field, or via the Wilson line of the gauge field around $x^{5}$. Here we use the latter. Then the partition function is

$$
Z_{5 d}=\operatorname{tr}(-1)^{F} \exp \left[i \beta H+\beta\left(\epsilon_{1} J_{1}+\epsilon_{2} J_{2}+a_{i} H^{i}\right)\right]
$$

where $H$ is the Hamiltonian, $J_{1,2}$ are the rotations of $z_{1,2}$-planes corrected with an appropriate amount of the $\mathrm{SU}(2)$ R-symmetry to commute with the supercharge, and $H^{i}$ are the generators of the Cartan of the gauge group. Here the trace is taken in the field theory Hilbert space. 3

\footnotetext{
${ }^{3}$ Physically, it would be more natural to take $\epsilon_{1,2}$ and $a_{i}$ to be purely imaginary, but supersymmetry guarantees that $Z$ is holomorphic with respect to them. For convenience we regard $\epsilon_{1,2}$ as real and $a_{i}$ as purely imaginary.
} 
Using the localization, the partition function can be written as the product of the oneloop contribution and the instanton contribution. The contribution $Z_{k}$ from $k$-instanton configurations is

$$
Z_{k, 5 d}=\operatorname{tr}_{\mathcal{H}_{k, \mathrm{BPS}}} \exp \beta\left(\epsilon_{1} J_{1}+\epsilon_{2} J_{2}+a_{i} H^{i}\right)
$$

where $\mathcal{H}_{k, \mathrm{BPS}}$ is the BPS subspace of the Hilbert space of the supersymmetric quantum mechanics from the $k$-instanton configurations. For the pure gauge theory, this is just the supersymmetric sigma model whose target space is the $k$-instanton moduli space $\mathcal{M}_{G, k}$ of gauge group $G$. Then the BPS subspace $\mathcal{H}_{k \text {,BPS }}$ is the space of holomorphic functions on $\mathcal{M}_{G, k}$. Therefore $Z_{k, 5 d}$ is just the character of the holomorphic functions on $\mathcal{M}_{G, k}$ under the action of the spacetime rotation $\mathrm{U}(1)^{2}$ and the gauge rotation $G$. This quantity is also known as the Hilbert series.

In the $\beta \rightarrow 0$ limit, $Z_{k, 5 d}$ is known to behave as

$$
Z_{k, 5 d} \sim \beta^{-2 k h^{\vee}} Z_{k}\left(\boldsymbol{a} ; \epsilon_{1,2}\right)
$$

and then the $4 \mathrm{~d}$ instanton partition function is given by

$$
Z_{\text {inst }}=\sum_{k} \Lambda^{2 k h^{\vee}} Z_{k}\left(\boldsymbol{a} ; \epsilon_{1,2}\right) .
$$

Then the instanton part of the prepotential is given by

$$
F_{\text {inst }}=\lim _{\epsilon_{1}, \epsilon_{2} \rightarrow 0} \epsilon_{1} \epsilon_{2} \log Z_{\text {inst }} .
$$

\subsection{One-instanton contribution}

Let us calculate the one-instanton contribution for arbitrary $G$. When $G$ is classical, we can use the ADHM description of the instanton moduli space to obtain the contribution [49, 50, 19]. Here, we use a more direct approach.

The one-instanton configuration of arbitrary gauge group $G$ is obtained by embedding an $\mathrm{SU}(2)$ BPST instanton into $G$ via a map $\mathrm{SU}(2) \hookrightarrow G$ associated to the long root of $G$ [51, 52, 53, 54, 55]. Therefore the one-instanton moduli space has a decomposition

$$
\mathcal{M}_{G, 1}=\mathbb{C}^{2} \times \tilde{\mathcal{M}}_{G, 1}
$$

where the factor $\mathbb{C}^{2}$ stands for the center of the instanton, and $\tilde{\mathcal{M}}_{G, 1}$ stands for the size and the gauge direction of the instanton. $\mathcal{M}_{G, 1}$ is a hyperkähler cone of real dimension $4\left(h^{\vee}-1\right)$.

Holomorphic functions on $\mathbb{C}^{2}$ are just polynomials of the coordinates $z_{1}$ and $z_{2}$, and the character under the rotations $\mathrm{U}(1)^{2} \subset \mathrm{SO}(4)$ is just $\left(1-e^{\beta \epsilon_{1}}\right)^{-1}\left(1-e^{\beta \epsilon_{2}}\right)^{-1}$. The space of holomorphic functions on $\tilde{\mathcal{M}}_{G, 1}$ was known to mathematicians e.g. [24, 25] using the fact that $\tilde{\mathcal{M}}_{G, 1}$ is the orbit of the highest weight vector in $\mathfrak{g}_{\mathbb{C}}$ under $G_{\mathbb{C}}$. The same space was also 
studied from a physical point of view by [26]. The conclusion is that as the representation of $\mathrm{U}(1)^{2} \times G$, the space of the holomorphic functions on $\mathcal{M}_{G, 1}$ is decomposed as

$$
\bigoplus_{m}\left(T_{1} T_{2}\right)^{\otimes m} \otimes V\left(-m \boldsymbol{\alpha}_{0}\right)
$$

where $T_{i}$ is the 1 -dimensional representation of $\mathrm{U}(1)$ with character $e^{\beta \epsilon_{i}}, V(\boldsymbol{w})$ is the irreducible representation of $G$ of the highest weight $\boldsymbol{w}$, and $-\boldsymbol{\alpha}_{0}$ is the highest root of the root system of $G$. The factor $\left(T_{1} T_{2}\right)^{\otimes m}$ arises from the fact that the radial direction is generated by $e^{\left(\epsilon_{1}+\epsilon_{2}\right) / 2}$ under the same $\mathrm{U}(1)^{2} \subset \mathrm{SO}(4)$.

Using the Weyl character formula, the character of this representation can be expressed as a summation over Weyl group elements, which can then be simplified as a summation over long roots, as we explain in Appendix $\mathrm{B}$. The end result is that the $4 \mathrm{~d} 1$-instanton contribution, including the contribution from the centre of mass, is given by

$$
Z_{k=1}=-\frac{1}{\epsilon_{1} \epsilon_{2}} \sum_{\boldsymbol{\gamma} \in \Delta_{l}} \frac{1}{\left(\epsilon_{1}+\epsilon_{2}+\boldsymbol{\gamma} \cdot \boldsymbol{a}\right)(\boldsymbol{\gamma} \cdot \boldsymbol{a}) \prod_{\boldsymbol{\gamma}^{\vee} \cdot \boldsymbol{\alpha}=1, \boldsymbol{\alpha} \in \Delta}(\boldsymbol{\alpha} \cdot \boldsymbol{a})},
$$

where $\Delta$ and $\Delta_{l}$ are the sets of the roots and the long roots, respectively. The one-instanton contribution to the prepotential via (2.6) reproduces the instanton calculation by Ito and Sasakura [56]. Explicit results for individual $G$ will be discussed in Sec. 4.3.

\section{Free field realization of $\mathrm{W}$-algebras}

We will construct our W-algebras of type $\Gamma=A_{n}, D_{n}$ and $E_{n}$ from free fields using the quantum version [27, 28] of the Drinfeld-Sokolov reduction [57]. For our purpose this boils down to the following steps: We introduce the free bosons $\boldsymbol{\varphi}$ living in the weight space of the semisimple Lie group $\Gamma$ of rank $n$. We normalize the OPE of the free bosons so that $J^{k}=i \partial \varphi^{i}$ satisfies

$$
J^{k}(z) J^{l}(w) \sim \frac{\delta^{k l}}{(z-w)^{2}} .
$$

We assume $\Gamma$ is simply-laced, and normalize the roots to have squared length 2 . The $\mathrm{W}$ algebra is then given by the centralizer of the screening charges $Q_{i}^{ \pm}$defined as follows: For each simple root $\boldsymbol{\alpha}_{i}$ there are charges

$$
Q_{i}^{ \pm}=\oint \tilde{s}_{i}^{ \pm} d z, \quad \tilde{s}_{i}^{ \pm}=\exp \left(b^{ \pm 1} \boldsymbol{\alpha}_{i} \cdot \boldsymbol{\varphi}\right) .
$$

Then we find the operators constructed from the bosons $\boldsymbol{\varphi}$ which commute with all the screening charges. It is known that there are $n$ independent generators with weights $w_{i}$, tabulated in Table. 1. The weight 2 operator is the energy-momentum tensor given by

$$
T(z)=W^{(2)}(z)=-\frac{1}{2}(\partial \boldsymbol{\varphi} \cdot \partial \boldsymbol{\varphi})(z)+Q \boldsymbol{\rho} \cdot \partial^{2} \boldsymbol{\varphi}(z),
$$


where $\boldsymbol{\rho}$ is the Weyl vector and $Q=b+1 / b$.

Our computations were all done in Mathematica using the package OPEdef s.m developed by K. Thielemans [30]. For more details on W-algebras, the readers should refer to the review [29]. In the following, all composite operators are understood to be OPE-normal ordered.

\subsection{Simply laced W-algebras}

\subsection{1 $A_{n}$}

For $A_{n}$ we make use of the quantum Miura transform [58, 59]. Let $\boldsymbol{e}_{i}, i=1, \ldots n+1$ be the weights of the fundamental $n+1$ dimensional representation of $A_{n}$, as in Appendix $\mathrm{A}$. We then construct a set of generators $U^{(k)}(z)$ from

$$
R^{(n+1)}(z)=-\sum_{k=0}^{n+1} U^{(k)}(z)(Q \partial)^{n+1-k}=\left(Q \partial-\boldsymbol{e}_{1} \cdot \partial \boldsymbol{\varphi}(z)\right) \cdots\left(Q \partial-\boldsymbol{e}_{n+1} \cdot \partial \boldsymbol{\varphi}(z)\right) .
$$

One can show that the singular part of the OPE of $R^{(n+1)}$ with $\tilde{s}_{i}^{ \pm}$is a total derivative, which means that the $U^{(k)}(z)$ are indeed in the centralizer of the screening charges. Since $U^{(1)}(z)$ vanishes, the remaining set of generators has the correct dimensions $w_{i}$. One can also show that they are independent, from which we conclude that we have a full set of generators. Note however that these generators are certainly not unique, as we can always add suitable products and derivatives of lower order generators.

\subsection{2 $D_{n}$}

For $D_{n}$ we introduce $\boldsymbol{e}_{i}, i=1, \ldots n$ such that $\pm \boldsymbol{e}_{i}$ form the weights of the fundamental $2 n$ dimensional representation. We repeat the construction of $R^{(n)}(z)$ as

$$
R^{(n)}(z)=-\sum_{k=0}^{n} V^{(k)}(z)(Q \partial)^{n-k}=\left(Q \partial-\boldsymbol{e}_{1} \cdot i \partial \boldsymbol{\varphi}(z)\right) \cdots\left(Q \partial-\boldsymbol{e}_{n} \cdot i \partial \boldsymbol{\varphi}(z)\right) .
$$

In this case however it turns out that only $V^{(n)}(z)$ commutes with the screening charges. To obtain the rest of the generators, we can take the OPE of $V^{(n)}$ with itself,

$$
V^{(n)}(z) V^{(n)}(w)=\frac{a_{n}}{(z-w)^{2 n}}+\sum_{k=1}^{n-1} \frac{a_{n-k}}{(z-w)^{2(n-k)}}\left(U^{(2 k)}(z)+U^{(2 k)}(w)\right),
$$

where we choose the normalization $a_{k}=\prod_{j=1}^{k-1}\left(1-2 j(2 j+1) Q^{2}\right)$. Once again one can show that $V^{(n)}$ and the $U^{(2 k)}$ are independent, which gives us a set of $n$ generators of the correct weights [60, 61]. 


\subsection{3 $E_{n}$}

For W-algebras of type $E_{n}$, the concise Miura transforms such as (3.4) for type $A_{n}$ and (3.5) for type $D_{n}$ are not known. Thus, one is forced to construct the commutants of the screening operators (3.2) directly. Note that an operator $O(z)$ commute with the screening charge $Q_{i}$ if and only if it has the form

$$
O(z)=\sum_{a} X_{a}\left(T_{j}\right)(z) Y_{a}\left(\partial \varphi^{1}, \ldots, \partial \varphi^{i-1}, \partial \varphi^{i+1}, \ldots, \partial \varphi^{n}\right)(z)
$$

where

- $X_{a}$ and $Y_{a}$ stand for normal-ordered polynomials, possibly with derivatives, constructed from their respective arguments,

- $T_{i}$ is the energy momentum tensor for the boson $\varphi_{i}=\boldsymbol{\alpha}_{i} \cdot \boldsymbol{\varphi}$ along the root $\boldsymbol{\alpha}_{i}$, i.e. $T_{i}(z)=-\partial \varphi_{i} \partial \varphi_{i}(z)+Q \partial^{2} \varphi_{i} / 2$ with central charge $1+6 Q^{2}$,

- and $\varphi^{i}=\boldsymbol{w}^{i} \cdot \boldsymbol{\varphi}$ where $\boldsymbol{w}^{i}$ are the fundamental weights, so that $Y_{a}$ are constructed from bosons perpendicular to the root $\boldsymbol{\alpha}_{i}$.

Therefore, $O(z)$ is in the W-algebra of type $E_{n}$ if and only if $O(z)$ has the decomposition (3.7) for each simple root $\boldsymbol{\alpha}_{j}$. Details of the construction of the $W\left(E_{6}\right)$ algebra are presented in Appendix D. The authors did not attempt to construct W-algebras of type $E_{7,8}$.

\subsection{Twisted sectors of the simply-laced W-algebras}

We need to consider the sectors of the simply-laced algebras twisted by their outer automorphisms to compare with the instanton partition function for non-simply-laced gauge groups 4

The $\mathbb{Z}_{r}$ outer automorphism acts on the simple roots as shown in Fig. 1. This induces an action on the free bosons $\varphi$. Since this is also a symmetry of the $\mathrm{W}$-algebra, and we can consider a $\mathbb{Z}_{r}$-twisted state. In practice we pick new linear combinations of bosons $\tilde{\varphi}_{j}$ which are eigenstates of the $\mathbb{Z}_{r}$ action:

$$
\partial \tilde{\varphi}_{j} \mapsto e^{2 \pi i k_{j} / r} \partial \tilde{\varphi}_{j}, \quad k_{j}=0, \ldots r-1 .
$$

Their modes are therefore in $\mathbb{Z}+k_{j} / r$. The set of generators $W^{\left(w_{i}\right)}$ therefore decompose into generators $W^{\left(\hat{w}_{i}\right)}$ with integer modes, and generators $W^{\left(\tilde{w}_{i}\right)}$ with non-integer modes. The former correspond to the invariants of the non-simply-laced gauge group that we want to construct, and the states of the lowest level in the twisted Verma module is generated by $W_{-1 / r}^{\left(\tilde{w}_{i}\right)}$.

The actions on the $\mathrm{W}$-generators are given explicitly as follows:

\footnotetext{
${ }^{4}$ The $\mathrm{W}$-algebras for non-simply-laced groups $B_{n}, C_{n}, G_{2}, F_{4}$ can also be determined via Drinfeld-Sokolov reduction, see e.g. [62, but that is not what we use.
} 
- The $\mathbb{Z}_{2}$ action on $W\left(A_{n}\right)$ maps $\tilde{U}^{(k)} \rightarrow(-1)^{k} \tilde{U}^{(k)}$ where $\tilde{U}^{(k)}$ is a suitable redefinition of $U^{(k)}$ defined in (3.4) . For example, in the case of $W\left(A_{5}\right)$,

$$
\begin{array}{rlrl}
\tilde{U}^{(2)} & =U^{(2)}, & \tilde{U}^{(3)}=U^{(3)}-2 Q \partial \tilde{U}^{(2)}, \\
\tilde{U}^{(4)}=U^{(4)}-\frac{3}{2} Q \partial \tilde{U}^{(3)}, & \tilde{U}^{(5)}=U^{(5)}-Q \partial \tilde{U}^{(4)}+Q^{3} \partial^{3} \tilde{U}^{(2)}, \\
\tilde{U}^{(6)}=U^{(6)}-\frac{1}{2} Q \partial \tilde{U}^{(5)}+\frac{1}{4} Q^{3} \partial^{3} \tilde{U}^{(3)} . &
\end{array}
$$

The redefined currents are determined by requiring $\mathrm{W}$-generators to have definite $Z_{2}$ eigenvalues.

- The $\mathbb{Z}_{2}$ action on $W\left(D_{n}\right)$ maps $V^{(n)} \rightarrow-V^{(n)}, U^{(2 k)} \rightarrow U^{(2 k)}$ where $V^{(n)}$ and $U^{(2 k)}$ are defined in (3.5), (3.6).

- The $\mathbb{Z}_{2}$ action on $W\left(E_{6}\right)$ maps $W^{(n)} \rightarrow(-1)^{n} W^{(n)}$, where $W^{(n)}$ is defined in Appendix D.

- The $\mathbb{Z}_{3}$ action on $W\left(D_{4}\right)$ is given is induced from the $\mathbb{Z}_{3}$ action on the four free bosons (3.8). Explicitly, we first define $W^{(2,4,6)}$ and $\tilde{W}^{(4)}$ by

$$
\begin{array}{ll}
W^{(2)}=U^{(2)}, & W^{(4)}=U^{(4)}+\frac{1}{4} \partial^{2} W^{(2)}-\frac{1}{2} U^{(4)} W^{(2)}+\frac{9 Q^{2}\left(1-4 Q^{2}\right)}{2-12 Q^{2}} \partial^{2} W^{(2)}, \\
\tilde{W}^{(4)}=\sqrt{3} V^{(4)}, & W^{(6)}=U^{(6)}+\frac{1}{2} \partial^{2} W^{(4)}-\frac{1}{3} W^{(4)} W^{(2)}+\frac{5}{6} Q^{2} \partial^{2} W^{(4)} .
\end{array}
$$

where $V^{(4)}$ and $U^{(2,4,6)}$ are defined in (3.5), (3.6). Then $W^{(2,6)}$ is invariant and $W^{(4)}+$ $i \tilde{W}^{(4)} \rightarrow e^{2 \pi i / 3}\left(W^{(4)}+i \tilde{W}^{(4)}\right)$ under the $\mathbb{Z}_{3}$ action.

\subsection{Basic properties of the Verma module}

Before continuing, we recall two basic features of the untwisted and twisted Verma module. The first is their Weyl invariance. The zero modes of the $\mathrm{W}$-generators are given in terms of the zero modes $\boldsymbol{J}_{0}$ of the free bosons $\boldsymbol{\varphi}$. If we define $\boldsymbol{a}$ by

$$
\boldsymbol{a}=\boldsymbol{J}_{0}-Q \boldsymbol{\rho}
$$

then the zero modes of $\mathrm{W}$-generators are Weyl-invariant polynomials of $\boldsymbol{a}$. For twisted sectors, the twisted bosons do not have zero modes. Correspondingly, $\boldsymbol{a}$ and $\boldsymbol{J}_{0}$ are invariant under the twist; $\boldsymbol{\rho}$ is automatically invariant.

The second is the Kac determinant at the lowest level, which we detail in Appendix C. Here we just quote the result, which is given by

$$
\text { (Kac determinant at level }-1 / r) \propto \prod_{\boldsymbol{\gamma} \in \Delta_{l}}(\boldsymbol{\gamma} \cdot \boldsymbol{a}+Q)
$$


where

$$
\Delta_{l}= \begin{cases}\Delta, & (r=1) \\ \{\boldsymbol{\alpha}+o(\boldsymbol{\alpha})\}, & (r=2) \\ \left\{\boldsymbol{\alpha}+o(\boldsymbol{\alpha})+o^{2}(\boldsymbol{\alpha})\right\} . & (r=3)\end{cases}
$$

Here $\boldsymbol{\alpha}$ runs over roots with $\boldsymbol{\alpha} \neq o(\boldsymbol{\alpha})$. As explained in Appendix A.2, $\Delta_{l}$ can be identified with the set of long roots of $G$, which is the S-dual of the $\mathbb{Z}_{r}$ invariant subgroup of $\Gamma$.

\section{Instantons and coherent states of W-algebras}

\subsection{Identification of the coherent state}

Under the correspondence of Nekrasov's partition functions and conformal blocks of Walgebras, the key relation is that the vev of the W-currents should become the fields $\phi^{\left(w_{i}\right)}(z)$ in the limit $\epsilon_{1,2} \ll a$ :

$$
\lim _{\epsilon_{1,2} \rightarrow 0}\left\langle W^{\left(w_{i}\right)}(z)\right\rangle(d z)^{w_{i}} \rightarrow \phi^{\left(w_{i}\right)}(z) .
$$

The fields $\phi^{\left(w_{i}\right)}(z)$ have two singularities $z=0, \infty$, which means that there is a state $\langle\mathcal{G}|$ at $z=\infty$ and a state $|\mathcal{G}\rangle$ at $z=0$. The behavior of $\phi^{\left(w_{i}\right)}(z)$ at $z=\infty$ has the same form as the behavior at $z=0$ by the map $w=1 / z$. Therefore the state $\langle\mathcal{G}|$ is a conjugate of the state $|\mathcal{G}\rangle$.

When $G$ is simply-laced, the conditions (1.1) and (1.2) imply that $|\mathcal{G}\rangle$ is in the Verma module of $W(G)$-algebra generated from the highest weight state $|\boldsymbol{w}\rangle$ with

$$
W_{0}^{\left(w_{i}\right)}|\boldsymbol{w}\rangle=w^{\left(w_{i}\right)}|\boldsymbol{w}\rangle
$$

where the eigenvalues $w^{\left(w_{i}\right)}$ should equal the vev $u^{\left(w_{i}\right)}$ up to some quantization error involving $\epsilon_{1,2}$. The condition (1.2) then tells us that

$$
W_{\ell}^{\left(w_{i}\right)}|\mathcal{G}\rangle=0 \quad \text { for } \ell>0 \text { unless } \quad W_{1}^{\left(h^{\vee}\right)}|\mathcal{G}\rangle=\Lambda^{h^{\vee}}|\mathcal{G}\rangle .
$$

When $G$ is non-simply-laced, the conditions (1.3) through (1.5) imply that $|\mathcal{G}\rangle$ is in the Verma module generated by the $\mathbb{Z}_{r}$-twisted vacuum $|\boldsymbol{w}\rangle$ of $W(\Gamma)$-algebra determined by

$$
W_{0}^{\left(\hat{w}_{i}\right)}|\boldsymbol{w}\rangle=w^{\left(\hat{w}_{i}\right)}|\boldsymbol{w}\rangle
$$

where the eigenvalues $w^{\left(\hat{w}_{i}\right)}$ should equal the vev $u^{\left(\hat{w}_{i}\right)}$ up to the quantization error involving $\epsilon_{1,2}$. The condition (1.5) then says

$$
W_{\ell}^{\left(w_{i}\right)}|\mathcal{G}\rangle=0 \quad \text { for } \ell>0 \text { unless } \quad W_{1 / r}^{\left(h^{\vee}\right)}|\mathcal{G}\rangle=\Lambda^{h^{\vee}}|\mathcal{G}\rangle .
$$

Then we should have the relation

$$
Z\left(\boldsymbol{a}, \epsilon_{1,2}\right)=\langle\mathcal{G} \mid \mathcal{G}\rangle .
$$


Both sides are power series; the $k$-instanton contribution on the left hand side corresponds to the level- $k / r$ contribution on the right hand side.

Note that the norm of $|\mathcal{G}\rangle$ does not change if we change the definition of $W^{\left(h^{\vee}\right)}$ by adding products and derivatives of lower degree generators, since $W_{-\ell}^{\left(h^{\vee}\right)}$ only changes by negative modes of lower generators, which annihilate $|\mathcal{G}\rangle$ anyway.

\subsection{Coherent state at the lowest level}

Here, we discuss the procedure to compute the norm of the Gaiotto-Whittaker vector $\langle\mathcal{G} \mid \mathcal{G}\rangle$ using the free-bosons representation of the W-algebras. The method is the same as in [22, 23]. We stick to the untwisted representations of the $\mathrm{W}$-algebras for the moment. Let us expand $|\mathcal{G}\rangle$ in terms of levels of descendants

$$
|\mathcal{G}\rangle=|\boldsymbol{w}\rangle+\Lambda^{h^{\vee}}\left|\mathcal{G}_{1}\right\rangle+\left(\Lambda^{h^{\vee}}\right)^{2}\left|\mathcal{G}_{2}\right\rangle+\cdots
$$

so that $\left|\mathcal{G}_{\ell}\right\rangle$ has conformal weight $\ell$. The condition (4.5) is now

$$
W_{\ell}^{\left(w_{i}\right)}\left|\mathcal{G}_{\ell}\right\rangle=0 \quad \text { for } n>0 \text { except for } \quad W_{1}^{\left(h^{\vee}\right)}\left|\mathcal{G}_{\ell}\right\rangle=\left|\mathcal{G}_{\ell-1}\right\rangle
$$

with $\left|\mathcal{G}_{0}\right\rangle=|\boldsymbol{w}\rangle$. In what follows, we will compute $\left\langle\mathcal{G}_{1} \mid \mathcal{G}_{1}\right\rangle$ and compare against the 1instanton computations. Expressed in terms of descendants at level $1\left|\mathcal{G}_{1}\right\rangle$ is

$$
\left|\mathcal{G}_{1}\right\rangle=\sum_{i} A_{i} W_{-1}^{\left(w_{i}\right)}|\boldsymbol{w}\rangle
$$

Now, use (4.8) to get

$$
\begin{aligned}
0 & =\left\langle\boldsymbol{w}\left|W_{1}^{\left(w_{i}\right)}\right| \mathcal{G}_{1}\right\rangle=\sum_{j} A_{j}\left\langle\boldsymbol{w}\left|W_{1}^{\left(w_{i}\right)} W_{-1}^{\left(w_{j}\right)}\right| \boldsymbol{w}\right\rangle=\sum_{j} K_{i j}^{(1)} A_{j} \text { for } w_{i} \neq h^{\vee} \\
1=\langle\boldsymbol{w} \mid \boldsymbol{w}\rangle & =\left\langle\boldsymbol{w}\left|W_{1}^{\left(h^{\vee}\right)}\right| \mathcal{G}_{1}\right\rangle=\sum_{j} A_{j}\left\langle\boldsymbol{w}\left|W_{1}^{\left(h^{\vee}\right)} W_{-1}^{\left(w_{j}\right)}\right| \boldsymbol{w}\right\rangle=\sum_{j} K_{n j}^{(1)} A_{j}
\end{aligned}
$$

where $K^{(\ell)}$ is the Kac-Shapovalov matrix at level $\ell$. We can solve for $A$ so that $A_{i}=\left(K^{(1)}\right)_{\text {in }}^{-1}$, and the norm is given by

$$
\left\langle\mathcal{G}_{1} \mid \mathcal{G}_{1}\right\rangle=\sum_{i, j} A_{i} K_{i j}^{(1)} A_{j}=\left(K^{(1)}\right)_{n n}^{-1}
$$

It can be easily generalized to arbitrary level to get $\left\langle\mathcal{G}_{\ell} \mid \mathcal{G}_{\ell}\right\rangle=\left(K^{(n)}\right)_{n^{\ell}, n^{\ell}}^{-1}$ where the index

$n^{\ell}$ means we pick the element corresponds to $\left(W_{-1}^{\left(w_{n}\right)}\right)^{\ell}|\boldsymbol{w}\rangle$. In order to get the norm of the Gaiotto states for the $\mathbb{Z}_{r}$ twisted sector, we simply look for the descendants of level-1/r, and take the corresponding element of the Kac-Shapovalov matrix.

To evaluate the Kac-Shapovalov matrix write the $W$ generators in terms of free bosons and expand as

$$
J^{k}(z)=i \partial \varphi^{k}(z)=\sum_{m \in \mathbb{Z}} J_{m}^{k} z^{-m-1}
$$


with the usual commutation relation

$$
\left[J_{m}^{k}, J_{n}^{l}\right]=m \delta^{k, l} \delta_{m+n, 0} .
$$

Then the W-algebra vacuum $|\boldsymbol{w}\rangle$ is represented by the free-boson vacuum $|\boldsymbol{a}\rangle$ where $\boldsymbol{a}=$ $\boldsymbol{J}_{0}-Q \boldsymbol{\rho}$ is the shifted zero mode of the free bosons. Note that the bra $\langle\boldsymbol{w}|$ then corresponds to $\langle-\boldsymbol{a}|$ due to the background charge $Q \boldsymbol{\rho}$ and the shift.

Let us first discuss the simply-laced case. As we are dealing with normal ordered products, the descendant states at level one can be expressed as

$$
W_{-1}^{\left(w_{i}\right)}|\boldsymbol{a}\rangle=\sum_{j} M_{i j}(\boldsymbol{a}) J_{-1}^{j}|\boldsymbol{a}\rangle, \quad\langle-\boldsymbol{a}| W_{1}^{\left(w_{i}\right)}=\sum_{j}\langle-\boldsymbol{a}| J_{1}^{i} M_{i j}(-\boldsymbol{a}) .
$$

The coefficient $M_{i j}(\boldsymbol{a})$ is a polynomial in $\boldsymbol{a}$ and $Q$. The Kac-Shapovalov matrix is given by

$$
K_{i j}(\boldsymbol{a})=\sum_{k} M_{i k}(-\boldsymbol{a}) M_{k j}(\boldsymbol{a}) .
$$

The twisted case is slightly more involved. Again we know that the lowest descendant states can be written as

$$
W_{-1 / r}^{\left(\tilde{w}_{i}\right)}|\boldsymbol{a}\rangle=\sum_{j} \tilde{M}_{i j}(\boldsymbol{a}) J_{-1 / r}^{j}|\boldsymbol{a}\rangle,
$$

where $\tilde{M}_{i j}(\boldsymbol{a})$ is again a polynomial in the zero modes of the untwisted bosons. To compute it, we need to find modes of the form : $\left(J^{j}\right)^{m}:_{-1 / r}$, which can be found from the original prescription of OPE-normal ordering, that is by subtracting the singular part of the correlator of the $m$ bosons. For instance, to obtain the constant $C_{2 m+1}$ of the state $:\left(J^{j}\right)^{2 m+1}:_{-1 / 2}|\boldsymbol{a}\rangle=C_{2 m+1} J_{-1 / 2}|\boldsymbol{a}\rangle$ we extract the regular part of the correlator

$$
\left.\lim _{z_{i} \rightarrow z_{1}}\left\langle-\boldsymbol{a}\left|J_{1 / 2}^{j} J^{j}\left(z_{2 m+1}\right) J^{j}\left(z_{2 m}\right) \cdots J^{j}\left(z_{1}\right)\right| \boldsymbol{a}\right\rangle\right|_{\text {reg }}=\frac{C_{2 m+1}}{2 z_{1}^{2 m+1 / 2}},
$$

and similarly for the zero modes of even powers of $J$.

\subsection{Comparison}

After all these preparations, now we can compare the norm of the Gaiotto-Whittaker vector and the one-instanton partition function. Obviously, the norm of the Gaiotto-Whittaker vector can have poles only at the zero of the Kac determinant, (3.12), i.e. when $\boldsymbol{\beta} \cdot \boldsymbol{a}_{\mathrm{boson}}+Q=$ 0 for a long root $\beta$. We also have the formula of the one-instanton expression in the gauge theory side, (2.9), which has apparent poles when $\boldsymbol{\beta} \cdot \boldsymbol{a}_{\text {gauge }}=\epsilon_{1}+\epsilon_{2}$ for a long root $\beta$, or when $\boldsymbol{\gamma} \cdot \boldsymbol{a}_{\text {gauge }}=0$ for an arbitrary root $\gamma$. In order for them to have any chance of agreement, we need to identify

$$
\boldsymbol{a}_{\text {boson }}=\frac{\boldsymbol{a}_{\text {gauge }}}{\sqrt{\epsilon_{1} \epsilon_{2}}}, \quad Q=\frac{\epsilon_{1}+\epsilon_{2}}{\sqrt{\epsilon_{1} \epsilon_{2}}} .
$$

Using the procedure outlined above, we have checked the agreement between the norm of the coherent state and instanton partition function at 1-instanton level 
- for simply-laced algebras $A_{1,2,3,4,5,6}, D_{4}$, and $E_{6}$,

- and for non-simply-laced algebras $B_{2,3}, C_{n}, F_{4}$ and $G_{2}$.

In general, the agreement comes with a multiplicative ambiguity due to the normalization of $\mathrm{W}$-currents. It can be easily absorbed into the redefinition of the expansion parameter $\Lambda .5$ For higher rank algebras, such as $A_{5}, A_{6}, F_{4}$ and $E_{6}$, due to the computational complexity, we checked the agreement by plugging in several set of test numbers for the zero modes and $Q$ parameter instead of leaving it as a symbolic expression. Let us now discuss the cases $A_{n}, D_{n}, B_{n}, C_{n}, G_{2}$ and $F_{4}$ in this order.

\subsection{1 $A_{n}$}

The W-algebra calculation leads to the following explicit form of the Gaiotto-Whittaker vector at level one:

$$
\left|\mathcal{G}_{1}\right\rangle=\sum_{i} v_{i}(\boldsymbol{a}) J_{i,-1}|\boldsymbol{a}\rangle
$$

where

$$
v_{i}(\boldsymbol{a})=\sum_{j} C_{i j}(\boldsymbol{a}) w_{j}(\boldsymbol{a}), \quad w_{i}(\boldsymbol{a})=\frac{1}{\prod_{x<i}\left(a_{x}-a_{i}\right) \prod_{i<x}\left(Q-a_{i}+a_{x}\right)}
$$

where

$$
\begin{array}{llrl}
C_{i j}(\boldsymbol{a}) & =0, & & (i<j) \\
C_{i j}(\boldsymbol{a}) & =1, & & (i=j) \\
C_{i j}(\boldsymbol{a}) & =(-1)^{i-j} Q \frac{\prod_{j<k<i}\left(Q-a_{j}+a_{k}\right)}{\prod_{j<k \leq i}\left(a_{j}-a_{k}\right)} . & & (i>j)
\end{array}
$$

Here $a_{i}=\boldsymbol{e}_{i} \cdot \boldsymbol{a}$. We checked the validity for small $n$; we believe it is true in general.

The corresponding bra is given by

$$
\left\langle\mathcal{G}_{1}\right|=\sum_{i}\langle-\boldsymbol{a}| J_{i, 1} v_{i}(-\boldsymbol{a})
$$

Now, it can be checked that

$$
v_{i}(-\boldsymbol{a})=\sum_{j} \tilde{w}_{j}(\boldsymbol{a})\left(C^{-1}(\boldsymbol{a})\right)_{j i}, \text { with } \tilde{w}_{i}(\boldsymbol{a})=\frac{1}{\prod_{x<i}\left(Q-a_{x}+a_{i}\right) \prod_{i<x}\left(a_{i}-a_{x}\right)} .
$$

Therefore,

$$
\left\langle\mathcal{G}_{1} \mid \mathcal{G}_{1}\right\rangle=\sum_{i} \prod_{i \neq j} \frac{1}{\left(a_{i}-a_{j}\right)\left(Q-a_{i}+a_{j}\right)}
$$

\footnotetext{
${ }^{5}$ When the underlying gauge theory is conformal, one may encounter much more intricate map between expansion parameters. See [19, 20] for the details.
} 
which is indeed the one-instanton contribution of Nekrasov's partition function [63, 64, 23] calculated from the geometry of the one-instanton moduli space. For example,

$$
\begin{aligned}
Z_{\mathrm{SU}(2), 1} & =-\frac{2}{4 a^{2}-Q^{2}} \\
Z_{\mathrm{SU}(3), 1} & =\frac{6\left(a_{1}^{2}+a_{1} a_{2}+a_{2}^{2}-Q^{2}\right)}{\left[\left(2 a_{1}+a_{2}\right)^{2}-Q^{2}\right]\left[\left(a_{1}+2 a_{2}\right)^{2}-Q^{2}\right]\left[\left(a_{1}-a_{2}\right)^{2}-Q^{2}\right]} .
\end{aligned}
$$

Two comments are in order. First, note that the uniform formula (2.9) instead gives the following form

$$
Z_{k=1}=\sum_{i \neq j} \frac{1}{\left(a_{i}-a_{j}\right)\left(Q-a_{j}-a_{i}\right)} \prod_{k \neq i} \frac{1}{a_{i}-a_{k}} .
$$

The agreement of (4.27) and (4.30) are not easy to see, but they are equal nonetheless. Second, recall that each summand in the formula (4.27) comes from the contribution of a fixed point in the resolution of the one-instanton moduli space. Then the relation (4.21) means that the free boson basis $J_{-1}^{i}|\boldsymbol{a}\rangle$ is an upper-triangular redefinition of the basis formed by the fixed points, explicitly confirming the results of Maulik and Okounkov [65].

\subsection{2 $D_{n}$}

The $W\left(D_{n}\right)$ contains $\mathrm{W}$-generators of dimension $2,4, \cdots, 2 n-2$ and additionally of dimension $n$. The Nekrasov partition function for $D_{n}=S O(2 n)$ can be obtained easily by evaluating the contour integral expression in [49]. We have,

$$
Z_{S O(2 n), 1}=-\sum_{i=1}^{n}\left[\frac{\left(a_{i} \pm Q\right)\left(2 a_{i} \pm Q\right)}{\prod_{j \neq i}\left(a_{i}^{2}-a_{j}^{2}\right)\left(\left(a_{i} \pm Q\right)^{2}-a_{j}^{2}\right)}\right]
$$

where \pm means we sum over both signs and $a_{i}=\boldsymbol{e}_{i} \cdot \boldsymbol{a}$. We have checked the agreement up to $D_{4}$.

\subsection{3 $B_{n}$}

To get the coherent state for $B_{n}$, we start from $W\left(A_{2 n-1}\right)$. We need to evaluate the $n$ dimensional Kac-Shapovalov matrix, since all the odd-dimensional W-currents are twisted by $\mathbb{Z}_{2}$ automorphism. The $\mathbb{Z}_{2}$ action maps $\varphi_{i}$ to $-\varphi_{2 n+1-i}$. Then the eigenstates are

$$
\tilde{\varphi}_{i}^{+}=\varphi_{i}-\varphi_{2 n+1-i} \text { and } \tilde{\varphi}_{i}^{-}=\varphi_{i}+\varphi_{2 n+1-i}
$$

Then, the twisted $\mathrm{W}$-currents can be written as

$$
\tilde{U}_{-1 / 2}^{(m)}|\boldsymbol{a}\rangle=\sum_{i} B_{m, i}(\boldsymbol{a}) J_{-1 / 2}^{-, i}|\boldsymbol{a}\rangle,
$$


where $B_{m, i}$ is a function of zero modes of the untwisted free bosons. Now, we evaluate the Kac-Shapovalov matrix to obtain the norm of the coherent state. For example, when $n=3$,

$$
K_{B_{3}}=\left(\begin{array}{cc}
\left\langle-\boldsymbol{a}\left|\tilde{U}_{1 / 2}^{(3)} \tilde{U}_{-1 / 2}^{(3)}\right| \boldsymbol{a}\right\rangle & \left\langle-\boldsymbol{a}\left|\tilde{U}_{1 / 2}^{(3)} \tilde{U}_{-1 / 2}^{(5)}\right| \boldsymbol{a}\right\rangle \\
\left\langle-\boldsymbol{a}\left|\tilde{U}_{1 / 2}^{(5)} \tilde{U}_{-1 / 2}^{(3)}\right| \boldsymbol{a}\right\rangle & \left\langle-\boldsymbol{a}\left|\tilde{U}_{1 / 2}^{(5)} \tilde{U}_{-1 / 2}^{(5)}\right| \boldsymbol{a}\right\rangle
\end{array}\right)
$$

and take the inverse of $K_{B_{3}}$ and read off the $(2,2)$ component of it.

On the instanton side, we have,

$$
Z_{S O(2 n+1), 1}=\sum_{i=1}^{n}\left[\frac{\left(2 a_{i} \pm Q\right)}{a_{i} \prod_{j \neq i}\left(a_{i}^{2}-a_{j}^{2}\right)\left(\left(a_{i} \pm Q\right)^{2}-a_{j}^{2}\right)}\right],
$$

where \pm means that we sum over both signs and $a_{i}=\boldsymbol{\epsilon}_{i} \cdot \boldsymbol{a} / \sqrt{2}$. We find that they agree with the norm of the corresponding coherent states up to numeric constants.

\subsection{4 $C_{n}$}

The Gaiotto-Whittaker vector corresponds to $C_{n}$ can be obtained from the $W\left(D_{n+1}\right)$ algebra. We can just follow the same procedure as $B_{n}$ case, but in this case we can do much easily. There is only one $W\left(D_{n}\right)$-generator that is not invariant under $\mathbb{Z}_{2}$ which is $V^{(n)}$. In terms of free bosons, it only shifts the sign of one of the bosons. Therefore, the Kac-Shapovalov matrix is just a number, we can simply use (3.12). We get the norm of the Gaiotto-Whittaker vector to be

$$
\left\langle\mathcal{G}_{1} \mid \mathcal{G}_{1}\right\rangle \propto \frac{1}{\prod_{i=1}^{n}\left(Q^{2}-4 a_{i}^{2}\right)} .
$$

It is known that the moduli space (neglecting the centre of mass contribution) of one $\operatorname{Sp}(n)$ instanton is $\mathbb{C}^{2 n} / \mathbb{Z}_{2}$, and the corresponding Hilbert series is given by (3.32) of [26], whose $\beta \rightarrow 0$ limit can be easily taken. Or, equivalently, note that $\mathrm{U}(1)^{2} \times \mathrm{Sp}(n)$ acts on $\mathbb{C}^{2 n}$ with the eigenvalues $\left(\epsilon_{1}+\epsilon_{2}\right) / 2 \pm a_{i}$. Therefore the integral is just

$$
Z_{\mathrm{Sp}(n), 1}=\frac{1}{2} \frac{1}{\prod_{i=1}^{n}\left(Q^{2} / 4-a_{i}^{2}\right)}
$$

where $a_{i}=\boldsymbol{e}_{i} \cdot \boldsymbol{a}$ and the factor $1 / 2$ comes from the orbifolding. This expression can, of course, also be obtained from Formula (B.10). We see that they agree completely up to a multiplicative constant.

\subsection{5 $G_{2}$}

There is only one $W$-generator that has a $-1 / 3$ mode: $W^{\left(4, \frac{2}{3}\right)}=W^{(4)}+i \tilde{W}^{(4)}$. Therefore, the Kac-Shapovalov matrix is one-dimensional, which is given by

$$
\left\langle\boldsymbol{a}\left|W_{1 / 3}^{\left(4, \frac{2}{3}\right)} W_{-1 / 3}^{\left(4, \frac{2}{3}\right)}\right| \boldsymbol{a}\right\rangle \propto \frac{1}{4}\left(Q^{2}-6 a_{2}^{2}\right)\left[4 Q^{4}-12 Q^{2}\left(3 a_{1}^{2}+a_{2}^{2}\right)+9\left(3 a_{1}^{2}-a_{2}^{2}\right)^{2}\right] .
$$


We can use the formula (3.12) for this example as well. The norm of Gaiotto-Whittaker state is given as the inverse of the the above expression.

The Hilbert series was given in (5.48) and (5.49) of [26]. Taking the limit $\beta \rightarrow 0$, we obtain

$$
Z_{G_{2}, 1}=\frac{72}{\left(Q^{2}-6 a_{2}^{2}\right)\left[4 Q^{4}-12 Q^{2}\left(3 a_{1}^{2}+a_{2}^{2}\right)+9\left(3 a_{1}^{2}-a_{2}^{2}\right)^{2}\right]}
$$

in our variables 6 Here, $a_{i}=\boldsymbol{\epsilon}_{i} \cdot \boldsymbol{a}$. This expression can also be obtained from Formula (B.10). See the section A.2 for $G_{2}$ for explicit expression for the roots and their basis. We see that the Hilbert series result completely agrees with the norm of Gaiotto-Whittaker state up to a multiplicative constant.

\subsection{6 $F_{4}$}

The Kac-Shapovalov matrix we need to compute is

$$
K_{F_{4}}=\left(\begin{array}{cc}
\left\langle-\boldsymbol{a}\left|W_{1 / 2}^{(5)} W_{-1 / 2}^{(5)}\right| \boldsymbol{a}\right\rangle & \left\langle-\boldsymbol{a}\left|W_{1 / 2}^{(5)} W_{-1 / 2}^{(9)}\right| \boldsymbol{a}\right\rangle \\
\left\langle-\boldsymbol{a}\left|W_{1 / 2}^{(9)} W_{-1 / 2}^{(5)}\right| \boldsymbol{a}\right\rangle & \left\langle-\boldsymbol{a}\left|W_{1 / 2}^{(9)} W_{-1 / 2}^{(9)}\right| \boldsymbol{a}\right\rangle
\end{array}\right) .
$$

Honest computation of this matrix is too time consuming for a desktop computer of 2011, due to the complication in the evaluation of the normal ordering of twisted bosons in the expressions involving $W^{(9)}$ as in (4.18). Thankfully, the Kac determinant is known in closed form in (3.12). Therefore we find

$$
\left\langle\mathcal{G}_{1} \mid \mathcal{G}_{1}\right\rangle=\left\langle-\boldsymbol{a}\left|W_{1 / 2}^{(5)} W_{-1 / 2}^{(5)}\right| \boldsymbol{a}\right\rangle /(\text { Kac determinant }),
$$

which is fairly straightforward to compute. It was checked that it agrees with the instanton expression (2.9). The explicit results for $E_{6}$ and $F_{4}$ are too lengthy to put here. They can be found in the supplementary files.

\section{Acknowledgements}

The authors thank the initial wavefunction of the universe, which arranged so that NM and JS visited IPMU on the same week, without which this paper would not have been written. They also thank Kris Thielemans, who provided them the Mathematica package OPEdefs.m, without which the calculation was not possible. They also thank IPMU mathematicians Alexey Bondal and Scott Carnahan for their help in simplifying the Hilbert series into the closed form. They also thank Amihay Hanany, Lotte Hollands and Michael Kay for useful discussions. They also thank Nick Dorey for his advice on the correct usage of the English definite article the used in the title of the paper. NM and JS also thank the hospitality of IPMU during their visit.

\footnotetext{
${ }^{6}$ Here $\boldsymbol{a}_{\text {ours }}=\sqrt{3} \boldsymbol{a}_{\text {theirs }}$
} 
The work of CAK is supported by a John A. McCone Postdoctoral Fellowship. The work of NM is supported by a research grant of the Max Planck Society. This work is in addition supported in part by the DOE grant DE-FG03-92-ER40701. The work of YT is supported in part by World Premier International Research Center Initiative (WPI Initiative), MEXT, Japan through the Institute for the Physics and Mathematics of the Universe, the University of Tokyo.

\section{A Roots of simple Lie algebras}

Here we list the roots for all Lie algebras, emphasising how to embed the root space of a non-simply-laced algebra $G$ to that of a simply-laced algebra $\Gamma$. First let us present the simply-laced ones in detail. Note that the simple roots are named as in Figure 3 .

\section{A.1 Simply-laced algebras}

Roots of $A_{n}=\mathrm{SU}(n+1)$. We let $\left\{\boldsymbol{e}_{i}: 1 \leq i \leq n+1\right\}$ be an orthonormal basis. The positive roots are

$$
\Delta^{+}=\left\{\boldsymbol{e}_{i}-\boldsymbol{e}_{j}: 1 \leq i<j \leq n+1\right\}
$$

Note that the span of roots is only $n$-dimensional. The simple roots are

$$
\boldsymbol{\alpha}_{1}=\boldsymbol{e}_{1}-\boldsymbol{e}_{2}, \quad \boldsymbol{\alpha}_{2}=\boldsymbol{e}_{2}-\boldsymbol{e}_{3}, \quad \ldots, \quad \boldsymbol{\alpha}_{n}=\boldsymbol{e}_{n}-\boldsymbol{e}_{n+1}
$$

Roots of $D_{n}=\mathrm{SO}(2 n)$. The positive roots are

$$
\Delta^{+}=\left\{\boldsymbol{e}_{i} \pm \boldsymbol{e}_{j}: 1 \leq i<j \leq n\right\}
$$

The simple roots are

$$
\boldsymbol{\alpha}_{1}=\boldsymbol{e}_{1}-\boldsymbol{e}_{2}, \quad \boldsymbol{\alpha}_{2}=\boldsymbol{e}_{2}-\boldsymbol{e}_{3}, \quad \ldots, \quad \boldsymbol{\alpha}_{n-1}=\boldsymbol{e}_{n-1}-\boldsymbol{e}_{n}, \boldsymbol{\alpha}_{n}=\boldsymbol{e}_{n-1}+\boldsymbol{e}_{n}
$$

Roots of $E_{6}$. The 36 positive roots are

$$
\Delta^{+}=\left\{\boldsymbol{e}_{i}+\boldsymbol{e}_{j}, \boldsymbol{e}_{i}-\boldsymbol{e}_{j}\right\}_{i<j \leq 5} \cup\left\{\frac{1}{2}\left( \pm \boldsymbol{e}_{1} \pm \boldsymbol{e}_{2} \pm \boldsymbol{e}_{3} \pm \boldsymbol{e}_{4} \pm \boldsymbol{e}_{5}+\sqrt{3} \boldsymbol{e}_{6}\right)\right\}_{\# \text { minus signs even }}
$$

The simple roots are

$$
\begin{aligned}
\boldsymbol{\alpha}_{1} & =\frac{1}{2}\left(\boldsymbol{e}_{1}-\boldsymbol{e}_{2}-\boldsymbol{e}_{3}-\boldsymbol{e}_{4}-\boldsymbol{e}_{5}+\sqrt{3} e_{6}\right) \\
\boldsymbol{\alpha}_{i} & =\boldsymbol{e}_{i}-\boldsymbol{e}_{i-1}(i=2,3,4,5), \quad \boldsymbol{\alpha}_{6}=\boldsymbol{e}_{1}+\boldsymbol{e}_{2} .
\end{aligned}
$$



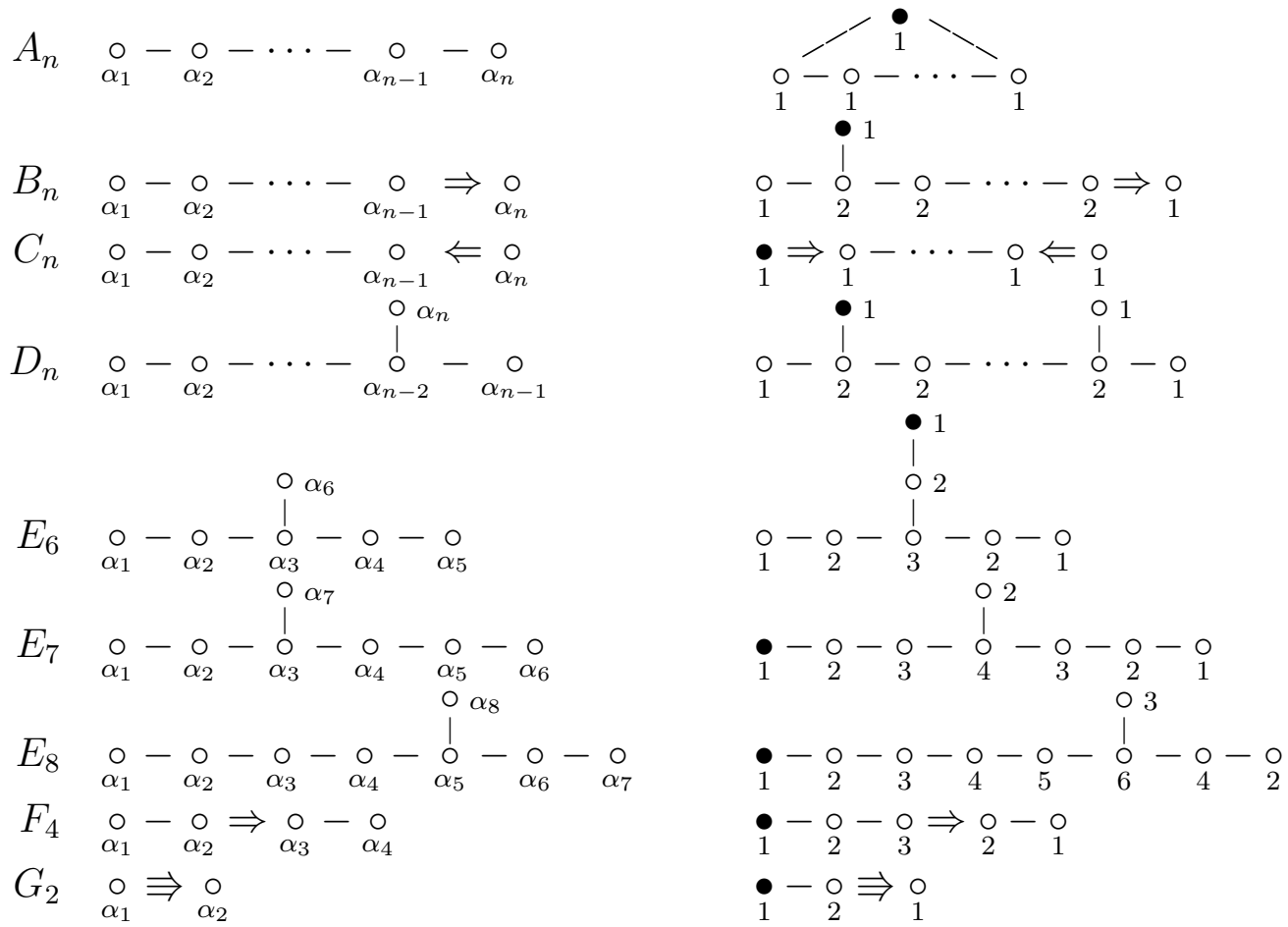

Figure 3: Dynkin diagrams of simple Lie algebras, our labeling of the simple roots, and the comarks. The extended node is shown by a black blob.

Roots of $E_{7}$. The 63 positive roots are

$$
\Delta^{+}=\left\{\boldsymbol{e}_{i}+\boldsymbol{e}_{j}, \boldsymbol{e}_{i}-\boldsymbol{e}_{j}\right\}_{i<j \leq 6} \cup\left\{\sqrt{2} \boldsymbol{e}_{7}\right\} \cup\left\{\frac{1}{2}\left( \pm \boldsymbol{e}_{1} \pm \ldots \pm \boldsymbol{e}_{6}+\sqrt{2} \boldsymbol{e}_{7}\right)\right\}_{\# \text { minus signs odd }}^{\text {(A. } 7)}
$$

Roots of $E_{8}$. The 120 positive roots are

$$
\Delta^{+}=\left\{\boldsymbol{e}_{i}+\boldsymbol{e}_{j}\right\}_{i<j \leq 8} \cup\left\{\boldsymbol{e}_{i}-\boldsymbol{e}_{j}\right\}_{i<j \leq 8} \cup\left\{\frac{1}{2}\left( \pm \boldsymbol{e}_{1} \pm \ldots \pm \boldsymbol{e}_{7}+\boldsymbol{e}_{8}\right)\right\}_{\# \text { minus signs even }}
$$

\section{A.2 Non-simply-laced algebras}

Let $\Gamma$ be a simply-laced algebra which is not $A_{2 n}$, and let $o$ be a symmetry of the Dynkin diagram. Note that $o$ can be viewed as an outer-automorphism acting on the Lie algebra of type $\Gamma$. It can be checked that $\alpha$ and $o(\alpha)$ are perpendicular when $\alpha \neq o(\alpha)$. Let us take

$$
\begin{aligned}
& \Delta_{s}=\{\boldsymbol{\alpha} \quad: \boldsymbol{\alpha} \in \Delta, \boldsymbol{\alpha}=o(\boldsymbol{\alpha})\}, \\
& \Delta_{l}=\{\boldsymbol{\alpha}+o(\boldsymbol{\alpha}) \quad: \boldsymbol{\alpha} \in \Delta, \boldsymbol{\alpha} \neq o(\boldsymbol{\alpha})\} \text { when } r=2, \\
& \Delta_{l}=\left\{\boldsymbol{\alpha}+o(\boldsymbol{\alpha})+o^{2}(\boldsymbol{\alpha}): \boldsymbol{\alpha} \in \Delta, \boldsymbol{\alpha} \neq o(\boldsymbol{\alpha})\right\} \text { when } r=3 \text {. }
\end{aligned}
$$


Then, $\Delta^{\prime}=\Delta_{s} \sqcup \Delta_{l}$ is a non-simply-laced root system, with $\Delta_{s}$ and $\Delta_{l}$ short and long roots respectively. In this normalization, the short root has length $\sqrt{2}$. Possible outerautomorphisms of simply-laced algebras that can be used are depicted in Figure 1. Every non-simply-laced algebra arises in this manner.

Note that one can also take the averages $\frac{1}{2}[\boldsymbol{\alpha}+o(\boldsymbol{\alpha})]$ and $\frac{1}{3}\left[\boldsymbol{\alpha}+o(\boldsymbol{\alpha})+o^{2}(\boldsymbol{\alpha})\right]$ in (A.10) and (A.11) respectively. A root system obtained this way corresponds to the subalgebra of $\Gamma$ invariant under the $\mathbb{Z}_{r}$ action, and is Langlands dual to the one obtained via (A.9)- A.11). It is natural in this latter convention for the long roots to have length $\sqrt{2}$. Even when the resulting root system is the same in two ways of folding, as in $G=G_{2}$ and $\Gamma=\mathrm{SO}(8)$, the embedding of the root space of $G$ into the root space of $\Gamma$ is different in the two cases. In this paper, we adhere to the former convention of folding. This convention is more natural in the context of singularity theory, see e.g. [38, 66]. Let us now go over the root systems of the non-simply-laced algebras one by one.

Roots of $B_{n}=\mathrm{SO}(2 n+1)$. The short positive roots and the long positive roots are

$$
\Delta_{s}^{+}=\left\{\boldsymbol{\epsilon}_{i},: 1 \leq i \leq n\right\}, \quad \Delta_{l}^{+}=\left\{\boldsymbol{\epsilon}_{i} \pm \boldsymbol{\epsilon}_{j}: 1 \leq i<j \leq n\right\}
$$

where $\boldsymbol{\epsilon}_{i} \cdot \boldsymbol{\epsilon}_{j}=2 \delta_{i j}$ so that the lengths of the short roots are $\sqrt{2}$.

This root system comes from a $\mathbb{Z}_{2}$ outer-automorphism $o$ of the root system of $A_{2 n-1}=$ $\mathrm{SU}(2 n)$ with the action mapping the simple root $\boldsymbol{\alpha}_{i}$ to $\boldsymbol{\alpha}_{2 n-i}$. The short and long positive roots of $B_{n}$ are then respectively

$$
\begin{aligned}
& \Delta_{s}^{+}=\left\{-\boldsymbol{e}_{i}+\boldsymbol{e}_{2 n+1-i}: i=1, \ldots, n\right\} \\
& \Delta_{l}^{+}=\left\{\left(-\boldsymbol{e}_{i}+\boldsymbol{e}_{2 n+1-i}\right) \pm\left(-\boldsymbol{e}_{j}+\boldsymbol{e}_{2 n+1-j}\right): 1 \leq i<j \leq n\right\}
\end{aligned}
$$

Setting

$$
\boldsymbol{\epsilon}_{i}=-\boldsymbol{e}_{i}+\boldsymbol{e}_{2 n+1-i}, \quad i=1, \ldots, n,
$$

the positive roots (A.13) and (A.14) become (A.12) as required.

Roots of $C_{n}=\operatorname{Sp}(n)$. The short positive roots and the long positive roots are

$$
\Delta_{s}^{+}=\left\{\boldsymbol{e}_{i} \pm \boldsymbol{e}_{j}: 1 \leq i<j \leq n\right\}, \quad \Delta_{l}^{+}=\left\{2 \boldsymbol{e}_{i}: 1 \leq i \leq n\right\}
$$

where $\boldsymbol{e}_{i} \cdot \boldsymbol{e}_{j}=\delta_{i j}$ so that the lengths of the short roots are $\sqrt{2}$.

This root system is obtained by applying a $\mathbb{Z}_{2}$ outer-automorphism to the root system of $D_{n+1}=\mathrm{SO}(2 n+2)$, which acts as follows:

$$
o: \boldsymbol{\alpha}_{n-1} \mapsto \boldsymbol{\alpha}_{n}, \quad \boldsymbol{\alpha}_{n} \mapsto \boldsymbol{\alpha}_{n-1}, \quad \boldsymbol{\alpha}_{i} \mapsto \boldsymbol{\alpha}_{i} \text { for } i \neq n, n-1
$$

From (A.4), it is clear that $o$ maps $\boldsymbol{e}_{n}$ to $-\boldsymbol{e}_{n}$ and leaves other $e_{i}$ invariant. Following the procedure, we easily get (A.16). 
Roots of $F_{4}$. The short positive roots are

$$
\Delta_{s}^{+}=\left\{\boldsymbol{\epsilon}_{i}\right\}_{i \leq 4} \cup\left\{\frac{1}{2}\left(\boldsymbol{\epsilon}_{1} \pm \boldsymbol{\epsilon}_{2} \pm \boldsymbol{\epsilon}_{3} \pm \boldsymbol{\epsilon}_{4}\right)\right\}
$$

and the long positive roots are

$$
\Delta_{l}^{+}=\left\{\boldsymbol{\epsilon}_{i}+\boldsymbol{\epsilon}_{j}\right\}_{i<j \leq 4} \cup\left\{\boldsymbol{\epsilon}_{i}-\boldsymbol{\epsilon}_{j}\right\}_{i<j \leq 4},
$$

where $\boldsymbol{\epsilon}_{i} \cdot \boldsymbol{\epsilon}_{j}=2 \delta_{i j}$ so that the length of the short roots is $\sqrt{2}$.

This root system can be obtained by applying a $\mathbb{Z}_{2}$ outer-automorphism to the root system of $E_{6}$ which maps $\left(\boldsymbol{\alpha}_{1}, \boldsymbol{\alpha}_{2}, \boldsymbol{\alpha}_{3}, \boldsymbol{\alpha}_{4}, \boldsymbol{\alpha}_{5}, \boldsymbol{\alpha}_{6}\right)$ to $\left(\boldsymbol{\alpha}_{5}, \boldsymbol{\alpha}_{4}, \boldsymbol{\alpha}_{3}, \boldsymbol{\alpha}_{2}, \boldsymbol{\alpha}_{1}, \boldsymbol{\alpha}_{6}\right)$. Therefore the simple roots of $F_{4}$ are given by

$$
\widehat{\boldsymbol{\alpha}}_{1}=\boldsymbol{\alpha}_{1}+\boldsymbol{\alpha}_{5}, \quad \widehat{\boldsymbol{\alpha}}_{2}=\boldsymbol{\alpha}_{2}+\boldsymbol{\alpha}_{4}, \quad \widehat{\boldsymbol{\alpha}}_{3}=\boldsymbol{\alpha}_{3}, \quad \widehat{\boldsymbol{\alpha}}_{4}=\boldsymbol{\alpha}_{6}
$$

We then introduce new basis $\boldsymbol{\epsilon}_{1,2,3,4}$ via

$$
\widehat{\boldsymbol{\alpha}_{1}}=\boldsymbol{\epsilon}_{2}-\boldsymbol{\epsilon}_{3}, \quad \widehat{\boldsymbol{\alpha}_{2}}=\boldsymbol{\epsilon}_{3}-\boldsymbol{\epsilon}_{4}, \quad \widehat{\boldsymbol{\alpha}_{3}}=\boldsymbol{\epsilon}_{4}, \quad \widehat{\boldsymbol{\alpha}_{4}}=\frac{1}{2}\left(\boldsymbol{\epsilon}_{1}-\boldsymbol{\epsilon}_{2}-\boldsymbol{\epsilon}_{3}-\boldsymbol{\epsilon}_{4}\right)
$$

These simple roots give rise to the positive roots listed in (A.18) and (A.19).

Roots of $G_{2}$. The short positive roots and long positive roots are

$$
\Delta_{s}^{+}=\left\{\sqrt{2} \epsilon_{1}, \pm \frac{1}{\sqrt{2}} \epsilon_{1}+\sqrt{\frac{3}{2}} \epsilon_{2}\right\}, \quad \Delta_{l}^{+}=\left\{ \pm \frac{3}{\sqrt{2}} \epsilon_{1}+\sqrt{\frac{3}{2}} \epsilon_{2}, \sqrt{6} \epsilon_{2}\right\}
$$

where $\boldsymbol{\epsilon}_{i} \cdot \boldsymbol{\epsilon}_{j}=\delta_{i j}$ so that the length of the short roots is $\sqrt{2}$.

This root system is obtained by applying a $\mathbb{Z}_{3}$ outer-automorphism to the root system of $D_{4}$, which maps the set of simple roots $\left(\boldsymbol{\alpha}_{1}, \boldsymbol{\alpha}_{2}, \boldsymbol{\alpha}_{3}, \boldsymbol{\alpha}_{4}\right)$ to $\left(\boldsymbol{\alpha}_{3}, \boldsymbol{\alpha}_{2}, \boldsymbol{\alpha}_{4}, \boldsymbol{\alpha}_{1}\right)$. The simple roots of $G_{2}$ is then

$$
\widehat{\alpha}_{1}=\boldsymbol{\alpha}_{1}+\boldsymbol{\alpha}_{3}+\boldsymbol{\alpha}_{4}, \quad \widehat{\alpha}_{2}=\boldsymbol{\alpha}_{2} .
$$

We then let

$$
\boldsymbol{\epsilon}_{1}=\frac{1}{\sqrt{2}}\left(\boldsymbol{e}_{1}-\boldsymbol{e}_{3}\right), \quad \boldsymbol{\epsilon}_{2}=\frac{1}{\sqrt{6}}\left(\boldsymbol{e}_{1}+2 \boldsymbol{e}_{2}-\boldsymbol{e}_{3}\right)
$$

resulting in $(\mathrm{A} .22$.

\section{B Hilbert series of the one-instanton moduli space}

In this appendix we derive expression (2.9) by computing the Hilbert series of the oneinstanton moduli space. Let $V(\boldsymbol{w})$ be the highest weight representation of $G$ of highest weight $\boldsymbol{w}$. As is conventional, we denote the highest root by $-\boldsymbol{\alpha}_{0}$, so that $V\left(-\boldsymbol{\alpha}_{0}\right)$ is the 


\begin{tabular}{r|rrr||r|rrr} 
& $|W|$ & $\left|\Delta_{l}\right|$ & $G_{0}$ & & $|W|$ & $\left|\Delta_{l}\right|$ & $G_{0}$ \\
\hline$A_{n}$ & $(n+1) !$ & $n^{2}+n$ & $A_{n-2}$ & $B_{n}$ & $2^{n} n !$ & $2 n(n-1)$ & $A_{1} \times B_{n-2}$ \\
$D_{n}$ & $2^{n-1} n !$ & $2 n(n-1)$ & $A_{1} \times D_{n-2}$ & $C_{n}$ & $2^{n} n !$ & $2 n$ & $C_{n-2}$ \\
$E_{6}$ & $72 \cdot 6 !$ & 72 & $A_{5}$ & $F_{4}$ & 1152 & 24 & $C_{3}$ \\
$E_{7}$ & $72 \cdot 8 !$ & 126 & $D_{6}$ & $G_{2}$ & 12 & 6 & $A_{1}$ \\
$E_{8}$ & $192 \cdot 10 !$ & 240 & $E_{7}$ & & & &
\end{tabular}

Table 2: Additional data of groups, required for the analysis in Appendix B.

adjoint representation. As explained in Sec. [2.2, the holomorphic function on the centred 1-instanton moduli space $\tilde{\mathcal{M}}_{G, 1}$ has the irreducible decomposition

$$
V=\bigoplus_{m=0}^{\infty} V\left(-m \boldsymbol{\alpha}_{0}\right) \otimes T^{\otimes m}
$$

under the action of $\mathrm{U}(1) \times G$, where $T$ is a one-dimensional representation of $\mathrm{U}(1)$. The character, or equivalently the Hilbert series is then

$$
Z=\operatorname{tr}_{V} e^{\phi} e^{\mu}
$$

where $\phi$ is an element of the Cartan subalgebra of $G$, and $e^{\mu}$ is the $\mathrm{U}(1)$ action. We will abbreviate $e^{\boldsymbol{\alpha} \cdot \boldsymbol{\phi}}$ as $e^{\alpha}$. The Weyl character formula then gives

$$
\begin{aligned}
Z & =\sum_{m=0}^{\infty} e^{m \mu} \frac{\sum_{w \in W} s(w) e^{-w m \boldsymbol{\alpha}_{0}+w \boldsymbol{\rho}}}{\prod_{\boldsymbol{\alpha} \in \Delta^{+}}\left(e^{\boldsymbol{\alpha} / 2}-e^{-\boldsymbol{\alpha} / 2}\right)}=\frac{\sum_{w \in W} s(w) e^{w \boldsymbol{\rho}} /\left(1-e^{\mu-w \boldsymbol{\alpha}_{0}}\right)}{\prod_{\boldsymbol{\alpha} \in \Delta^{+}}\left(e^{\boldsymbol{\alpha} / 2}-e^{-\boldsymbol{\alpha} / 2}\right)} \\
& =\sum_{\boldsymbol{\gamma} \in \Delta_{l}} \frac{1}{\left(1-e^{\mu+\boldsymbol{\gamma}}\right) \prod_{\boldsymbol{\alpha} \in \Delta^{+}}\left(e^{\boldsymbol{\alpha} / 2}-e^{-\boldsymbol{\alpha} / 2}\right)} \sum_{\substack{w \in W \\
-w \boldsymbol{\alpha}_{0}=\boldsymbol{\gamma}}} s(w) e^{w \boldsymbol{\rho}} .
\end{aligned}
$$

Here, $\Delta$ is the set of roots, $\Delta_{l}$ is the set of long roots, $\Delta^{+}$is the set of positive roots, $\rho$ is the Weyl vector, and $s(w)$ is the sign of an element $w$ of the Weyl group $W$.

Now, consider a subgroup $G_{0}$ of $G$, whose Dynkin diagram is formed by the nodes of the Dynkin diagram of $G$ which is not connected to the extended node of the affine Dynkin diagram, see Figure 3 and Table 2. The Weyl group of $G_{0}$ fixes $-\boldsymbol{\alpha}_{0}$ by construction. Furthermore, $|W(G)|=\left|\Delta_{l}\right| \cdot\left|W\left(G_{0}\right)\right|$. Therefore, $W\left(G_{0}\right)$ is exactly the subgroup of $W(G)$ which fixes $-\boldsymbol{\alpha}_{0}$.

The difference $\boldsymbol{\rho}(G)-\boldsymbol{\rho}\left(G_{0}\right)$ of the Weyl vectors of $G$ and $G_{0}$ is perpendicular to all $\boldsymbol{\alpha}_{i}$ of $G_{0}$, and therefore is proportional to $-\boldsymbol{\alpha}_{0}$. Therefore there is a constant $c$ such that

$$
\boldsymbol{\rho}(G)=-c \boldsymbol{\alpha}_{0}+\boldsymbol{\rho}\left(G_{0}\right) .
$$

To fix $c$, take the inner product with respect to $-\boldsymbol{\alpha}_{0}$, using the expansion $-\boldsymbol{\alpha}_{0}^{\vee}=\sum n_{i}^{\vee} \alpha_{i}^{\vee}$ where $n_{i}^{\vee}$ are the comarks shown in Figure 3. We find

$$
2 c=-\boldsymbol{\alpha}_{0}^{\vee} \cdot \boldsymbol{\rho}(G)=\sum n_{i}^{\vee} \boldsymbol{\alpha}_{i}^{\vee} \cdot \boldsymbol{\rho}(G)=\sum n_{i}^{\vee}=h^{\vee}-1 .
$$


Here we used that the sum of the comarks is $h^{\vee}-1$.

For a long root $\boldsymbol{\gamma}$, we pick a Weyl group element $w_{\boldsymbol{\gamma}}$ such that $-w_{\gamma} \boldsymbol{\alpha}_{0}=\boldsymbol{\gamma}$. The set of $w$ such that $-w \boldsymbol{\alpha}_{0}=\boldsymbol{\gamma}$ is then simply $w_{\gamma} W\left(G_{0}\right)$. Therefore,

$$
\begin{array}{r}
\sum_{-w \boldsymbol{\alpha}_{0}=\boldsymbol{\gamma}} s(w) e^{w \rho}=\sum_{w \in W\left(G_{0}\right)} s\left(w_{\boldsymbol{\gamma}} w\right) e^{w_{\boldsymbol{\gamma}} w \rho}=e^{-\left(h^{\vee}-1\right) w_{\boldsymbol{\gamma}} \boldsymbol{\alpha}_{0} / 2} s\left(w_{\boldsymbol{\gamma}}\right) \sum_{w \in W\left(G_{0}\right)} s(w) e^{w_{\boldsymbol{\gamma}} w \rho\left(G_{0}\right)} \\
=e^{\left(h^{\vee}-1\right) \gamma / 2} s\left(w_{\boldsymbol{\gamma}}\right) \prod_{\boldsymbol{\alpha} \in \Delta^{+}\left(G_{0}\right)}\left(e^{w_{\boldsymbol{\gamma}} \boldsymbol{\alpha} / 2}-e^{-w_{\boldsymbol{\gamma}} \boldsymbol{\alpha} / 2}\right) .
\end{array}
$$

Plugging it in to (B.3), we have

$$
\begin{aligned}
& Z=\sum_{\boldsymbol{\gamma} \in \Delta_{l}} \frac{e^{\left(h^{\vee}-1\right) \gamma / 2} s\left(w_{\boldsymbol{\gamma}}\right) \prod_{\boldsymbol{\alpha} \in \Delta^{+}\left(G_{0}\right)}\left(e^{w_{\boldsymbol{\gamma}} \boldsymbol{\alpha} / 2}-e^{-w_{\boldsymbol{\gamma}} \boldsymbol{\alpha} / 2}\right)}{\left(1-e^{\mu+\boldsymbol{\gamma}}\right) \prod_{\boldsymbol{\alpha} \in \Delta^{+}}\left(e^{\boldsymbol{\alpha} / 2}-e^{-\boldsymbol{\alpha} / 2}\right)} \\
& =\sum_{\boldsymbol{\gamma} \in \Delta_{l}} \frac{e^{\left(h^{\vee}-1\right) \gamma / 2} s\left(w_{\gamma}\right) \prod_{\boldsymbol{\alpha} \in \Delta^{+}\left(G_{0}\right)}\left(e^{w_{\gamma} \boldsymbol{\alpha} / 2}-e^{-w_{\gamma} \boldsymbol{\alpha} / 2}\right)}{\left(1-e^{\mu+\boldsymbol{\gamma}}\right) s\left(w_{\gamma}\right) \prod_{\boldsymbol{\alpha} \in \Delta^{+}}\left(e^{w_{\gamma} \boldsymbol{\alpha} / 2}-e^{-w_{\gamma} \boldsymbol{\alpha} / 2}\right)} \\
& =\sum_{\boldsymbol{\gamma} \in \Delta_{l}} \frac{e^{\left(h^{\vee}-1\right) \gamma / 2}}{\left(1-e^{\mu+\boldsymbol{\gamma}}\right) \prod_{\boldsymbol{\alpha} \in \Delta^{+} \backslash \Delta^{+}\left(G_{0}\right)}\left(e^{w_{\gamma} \boldsymbol{\alpha} / 2}-e^{-w_{\gamma} \boldsymbol{\alpha} / 2}\right)} .
\end{aligned}
$$

Recall that the inner product $-\boldsymbol{\alpha}_{0}^{\vee} \cdot \boldsymbol{\alpha}$ is

- 2 if and only if $\boldsymbol{\alpha}=-\boldsymbol{\alpha}_{0}$,

- 1 if and only if $\boldsymbol{\alpha} \in \Delta^{+} \backslash \Delta^{+}\left(G_{0}\right)$ and $\boldsymbol{\alpha} \neq-\boldsymbol{\alpha}_{0}$,

so that (B.7) can be further written as

$$
\begin{aligned}
& =\sum_{\boldsymbol{\gamma} \in \Delta_{l}} \frac{e^{\left(h^{\vee}-1\right) \gamma / 2}}{\left(1-e^{\mu+\gamma}\right)\left(e^{-w_{\gamma} \boldsymbol{\alpha}_{0} / 2}-e^{w_{\boldsymbol{\gamma}} \boldsymbol{\alpha}_{0} / 2}\right) \prod_{-\boldsymbol{\alpha}_{0}^{\vee} \cdot \boldsymbol{\alpha}=1}\left(e^{w_{\gamma} \boldsymbol{\alpha} / 2}-e^{-w_{\boldsymbol{\gamma}} \boldsymbol{\alpha} / 2}\right)} \\
& =\sum_{\boldsymbol{\gamma} \in \Delta_{l}} \frac{e^{\left(h^{\vee}-1\right) \gamma / 2}}{\left(1-e^{\mu+\gamma}\right)\left(e^{\gamma / 2}-e^{-\gamma / 2}\right) \prod_{\gamma^{\vee} \cdot \boldsymbol{\alpha}=1}\left(e^{\boldsymbol{\alpha} / 2}-e^{-\boldsymbol{\alpha} / 2}\right)} .
\end{aligned}
$$

Now the elements $w_{\gamma}$ is gone.

Note that $\left|\left\{\boldsymbol{\alpha} \mid \boldsymbol{\gamma}^{\vee} \cdot \boldsymbol{\alpha}=1\right\}\right|$ is $2 h^{\vee}-4$, so that (B.8) has $2 h^{\vee}-2$ terms in the denominator, which is the expected number for the centered instanton moduli space. To get the explicit expressions, we let

$$
e^{\boldsymbol{\alpha}} \equiv e^{\boldsymbol{\alpha} \cdot \phi}=e^{\beta \boldsymbol{\alpha} \cdot \boldsymbol{a}}, \quad e^{\mu}=e^{\beta\left(\epsilon_{1}+\epsilon_{2}\right)}
$$

In the papers on Hilbert series, e.g. [26], the variables $x_{i}=e^{a_{i}}$ were used instead. The $4 \mathrm{~d}$ version is obtained by taking the $\beta \rightarrow 0$ limit, giving

$$
\beta^{2 h^{\vee}-2} Z \rightarrow \sum_{\boldsymbol{\gamma} \in \Delta_{l}} \frac{-1}{\left(\epsilon_{1}+\epsilon_{2}+\boldsymbol{\gamma} \cdot \boldsymbol{a}\right)(\boldsymbol{\gamma} \cdot \boldsymbol{a}) \prod_{\boldsymbol{\gamma}^{\vee} \cdot \boldsymbol{\alpha}=1}(\boldsymbol{\alpha} \cdot \boldsymbol{a})} .
$$

Together with the contribution of $\mathbb{C}^{2}$ we indeed get (2.9) . 


\section{Kac determinant at the lowest level}

Here we consider the Kac determinant at the lowest level. The result for the untwisted case is well known, see e.g. [67, 29]. The twisted case also follows straightforwardly by modifying the derivation for the untwisted case. For example, the Kac determinant for the Drinfeld-Sokolov reduction with respect to the minimal nilpotent was determined in [68]. Here we need to perform it with respect to the principal nilpotent.

First, consider the untwisted Verma module. Let the zero modes of the free bosons be $\boldsymbol{J}_{0}$. Pick a simple root $\boldsymbol{\alpha}_{i}$, and let $\varphi_{i}=\boldsymbol{\alpha}_{i} \cdot \boldsymbol{\varphi}$. As explained in Sec. 3.1.3, elements in the W-algebra can be expanded in terms of the energy-momentum tensor $T_{i}$ for $\varphi_{i}$ and the free bosons perpendicular to $\varphi_{i}$. At level one, $\left(T_{i}\right)_{-1}=\left(\boldsymbol{\alpha}_{i} \cdot \boldsymbol{J}_{0}\right) J_{i,-1}$, and similarly, the $(-1)$ mode of any operator constructed out of $T_{i}$ comes with a factor of $\boldsymbol{\alpha}_{i} \cdot \boldsymbol{J}_{0}$. Thus, there is a null state in the $\mathrm{W}$-algebra Verma module when $\boldsymbol{\alpha}_{i} \cdot \boldsymbol{J}_{0}=0$. Therefore the Kac determinant at level one is divisible by $\boldsymbol{\alpha}_{i} \cdot \boldsymbol{J}_{0}=\boldsymbol{\alpha}_{i} \cdot \boldsymbol{a}+Q$. Due to the shifted Weyl invariance, the Kac determinant is divisible by $\boldsymbol{\alpha} \cdot \boldsymbol{a}+Q$ for all roots $\alpha$. The Kac determinant has degree $2 \sum\left(w_{i}-1\right)$ in $\boldsymbol{a}$, which equals the number of roots. It follows that

$$
(\text { Kac determinant at level } 1) \propto \prod_{\boldsymbol{\alpha} \in \Delta}(\boldsymbol{\alpha} \cdot \boldsymbol{a}+Q) .
$$

Next, consider the states at level $-1 / r$ of the $\mathbb{Z}_{r}$-twisted Verma module. Denote by $o$ the $\mathbb{Z}_{r}$ action, under which the zero mode $\boldsymbol{J}_{0}$ is invariant. We regard it as in the Cartan of $G$, the S-dual of the $\mathbb{Z}_{r}$-invariant subalgebra of $\Gamma$. Recall that the long simple roots $\gamma_{i}$ of $G$ are given by $\gamma_{i}=\boldsymbol{\alpha}_{i}+\boldsymbol{\alpha}_{o(i)}$ when $r=2$ and similarly for $r=3$, (A.10), (A.11).

Take a simple root such that $\boldsymbol{\alpha}_{i} \neq \boldsymbol{\alpha}_{o(i)}$. Assuming $\Gamma \neq A_{2 n}$, we have $\boldsymbol{\alpha}_{i} \cdot \boldsymbol{\alpha}_{o(i)}=0$. Therefore the operators in the $\mathrm{W}$-algebra can be written in terms of the energy-momentum tensors $T_{i}, T_{o(i)}$ (and $T_{o^{2}(i)}$ if $r=3$ ) for the free bosons $\varphi_{i}, \varphi_{o(i)}$ (and $\varphi_{o^{2}(i)}$ ) and free bosons perpendicular to $\varphi_{i}, \varphi_{o(i)}$ (and $\left.\varphi_{o^{2}(i)}\right)$. Now, the level $-(1 / r)$ states arise from $T_{i}-T_{o(i)}$ when $r=2$, and from $T_{i}+e^{2 \pi i / 3} T_{o(i)}+e^{4 \pi i / 3} T_{o^{2}(i)}$ when $r=3$. Recall that $T_{i} \propto-\left(\partial \varphi_{i} \partial \varphi_{i}\right)+Q \partial^{2} \varphi_{i} / 2$. Therefore the $(-1 / r)$-mode always arises with the combination

$$
\left(\boldsymbol{\alpha}_{i} \cdot \boldsymbol{J}_{0}-Q(1-1 / r)\right) J_{i,-1 / r}
$$

It follows the Kac determinant has a zero when $\boldsymbol{\alpha}_{i} \cdot \boldsymbol{J}_{0}=Q(1-1 / r)$ for a non-invariant simple root $\boldsymbol{\alpha}_{i}$, or in other words $\boldsymbol{\gamma}_{i} \cdot \boldsymbol{a}+Q=0$ for a long root $\boldsymbol{\gamma}_{i}$ and the shifted zero mode $\boldsymbol{a}$. From Weyl invariance, the Kac determinant has a factor $\boldsymbol{\gamma} \cdot \boldsymbol{a}+Q$ for each long root $\gamma$. The Kac determinant has degree $2 \sum_{i}\left(w_{i}-1\right)$ where the sum is over the degrees $w_{i}$ of $W(\Gamma)$-generators not invariant under $\mathbb{Z}_{r}$. This equals the number of long roots of $G$. We thus conclude that

$$
(\text { Kac determinant at level } 1 / r) \propto \prod_{\boldsymbol{\gamma} \in \Delta_{l}}(\boldsymbol{\gamma} \cdot \boldsymbol{a}+Q)
$$




\section{Construction of the $\mathrm{W}$-algebra of type $E_{6}$}

\section{D.1 Detailed procedure}

We provide some more details on the construction of the $W\left(E_{6}\right)$-algebra. We first construct the two lowest generators $W^{(2)}$ and $W^{(5)}$ utilizing $W\left(A_{5}\right)$ subalgebra. The higher generators $W^{(6,8,9,12)}$ we then obtain from suitable OPEs of $W^{(5)}$.

Let $J^{1, \ldots, 6}=i \partial \varphi^{1, \ldots, 6}$ be six orthonormal free bosons. Take the $A_{2} \times A_{2} \times A_{1}$ subalgebra of $E_{6}$, corresponding to the nodes of the Dynkin diagram except the central node. We can then choose the bosons in such a way that $J^{1,2}, J^{3,4}$ span the Cartan of the two $A_{2}$, and $J_{6}$ spans the Cartan of the $A_{1}$ respectively. Note that the $\mathbb{Z}_{2}$ outer automorphism exchanges the two $A_{2}$ subalgebras.

Consider now the $A_{5}$ subalgebra of $E_{6}$. It contains $A_{2} \times A_{2}$, but not the $A_{1}$ described above. Construct the generators $U_{2, \ldots, 5}(z)$ of the $W\left(A_{5}\right)$ algebra via (3.4), and obtain the $\mathbb{Z}_{2}$ eigenstates (3.9). We introduce the boson

$$
K(z)=\left(\sqrt{3} J^{5}+J^{6}\right) / 2 \sqrt{2},
$$

which is perpendicular to the Cartan of $A_{5}$. The most general ansatz for $W^{(2)}$ and $W^{(5)}$ compatible with $\mathbb{Z}_{2}$ is then

$$
\begin{aligned}
& W^{(2)}=U^{(2)}+c_{1} K^{2}+c_{2} \partial K, \\
& W^{(5)}=\tilde{U}^{(5)}+c_{3} \tilde{U}^{(3)} U^{(2)}+c_{4} \tilde{U}^{(3)} K^{2}+c_{5} \partial^{2} \tilde{U}^{(3)}+c_{6} \partial \tilde{U}^{(3)} K+c_{7} \tilde{U}^{(3)} \partial K .
\end{aligned}
$$

The constants $c_{1, \ldots, 7}$ can be determined by the method explained in section 3.1.3, we expand (D.3) in the $J^{i}$, and require that $J^{6}$ only appears in the form of its energy momentum tensor

$$
T(z)=-\left(J^{6}\right)^{2} / 2+(Q / \sqrt{2}) \partial J^{6}
$$

This fixes the constants to be

$$
c_{1}=1, c_{2}=-11 Q, c_{3}=0, c_{4}=1, c_{5}=\frac{3}{2} Q^{2}, c_{6}=-3 Q, c_{7}=2 Q .
$$

The higher generators can now be obtained by repeatedly taking the OPEs of $W^{(5)}$. Let $\left[O_{1} O_{2}\right]_{p}(w)$ be the coefficient of $(z-w)^{-p}$ of the OPE $O_{1}(z) O_{2}(w)$. We let

$$
\begin{aligned}
& W^{(6)}=\left(1+12 Q^{2}\right)^{-1}\left[W^{(5)} W^{(5)}\right]_{4}, \quad W^{(8)}=\left[W^{(5)} W^{(5)}\right]_{2}, \\
& W^{(9)}=\left[W^{(5)} W^{(6)}\right]_{2}, \quad W^{(12)}=\left[W^{(6)} W^{(8)}\right]_{2} \text {. }
\end{aligned}
$$

These OPEs a priori are not guaranteed to generate independent fields, but could contain just products of lower lying fields, possibly with derivatives. Independence can be confirmed as follows. Let $P^{(d)}$ be the terms in $W^{(d)}$ which do not contain derivatives, regarded as polynomials in $J^{i}$. It then suffices to check that they are algebraically independent, for 
which we can compute the Jacobian $\operatorname{det}\left(\partial P^{(d)} / \partial J^{i}\right)$ and check that it is non-zero. Note that W-generators constructed in this manner automatically have definite parity under $\mathbb{Z}_{2}$.

Unfortunately these OPE calculations, when expressed in terms of six free bosons, are too much for a laptop computer of 2011. It is thus necessary to use the $A_{2} \times A_{2} \times A_{1}$ subalgebra to organize the computation. The generator of $W\left(A_{1}\right)$ is $T(z)$ in (D.4). Let $U^{(2,3)}$ be the generators of the first $W\left(A_{2}\right)$, and $\tilde{U}^{(2,3)}$ be those of the second, as defined in (3.4). Then, we can rewrite $W^{(2)}$ and $W^{(5)}$ determined in $(\overline{\mathrm{D} .3)})$ in terms of $U^{(2,3)}, \tilde{U}^{(2,3)}$, $J_{5}$, and $T$. Now the OPEs in (D.7) can be and were performed using the known OPEs of $W\left(A_{2}\right) \times W\left(A_{2}\right) \times W\left(A_{1}\right)$ in about three hours on a $2 \mathrm{GHz}$ machine with 3 Gbytes of memory; the independence of the resulting generators can be and was checked. The denominator $\left(1+12 Q^{2}\right)$ for $W^{(6)}$ in (D.7) is inserted because the OPE turns out to be divisible by this factor.

\section{D.2 Explicit generators}

In the following, we list the generators thus constructed; we set $J=J_{5}, A_{i}=U^{(i)}+\tilde{U}^{(i)}$ and $B_{i}=U^{(i)}-\tilde{U}^{(i)}$. With explicit generators, it is easy to check that the level-1 contribution to the norm of the coherent state as calculated by (4.12) is equal to the 1-instanton result (2.9), once when the zero modes are substituted with random numbers.

\section{D.2.1 $W_{2}$}

D.2.2 $W_{5}$

D.2.3 $W_{6}$

D.2.4 $W_{8}$

\section{D.2.5 $W_{9}$}

D.2.6 $\quad W_{12}$ 


\section{References}

[1] N. Seiberg and E. Witten, "Monopole Condensation, and Confinement in $\mathcal{N}=2$ Supersymmetric Yang-Mills Theory," Nucl. Phys. B426 (1994) 19-52, arXiv:hep-th/9407087.

[2] N. Seiberg and E. Witten, "Monopoles, Duality and Chiral Symmetry Breaking in $\mathcal{N}=2$ Supersymmetric QCD," Nucl. Phys. B431 (1994) 484-550, arXiv:hep-th/9408099.

[3] A. Klemm, W. Lerche, P. Mayr, C. Vafa, and N. P. Warner, "Self-Dual Strings and $\mathcal{N}=2$ Supersymmetric Field Theory," Nucl. Phys. B477 (1996) 746-766, arXiv: hep-th/9604034.

[4] E. Witten, "Solutions of Four-Dimensional Field Theories via M-theory," Nucl. Phys. B500 (1997) 3-42, arXiv:hep-th/9703166.

[5] D. Gaiotto, "N=2 Dualities," arXiv:0904.2715 [hep-th].

[6] D. Gaiotto, G. W. Moore, and A. Neitzke, "Wall-Crossing, Hitchin Systems, and the WKB Approximation," arXiv:0907.3987 [hep-th].

[7] A. Klemm, M. Mariño, and S. Theisen, "Gravitational Corrections in Supersymmetric Gauge Theory and Matrix Models," JHEP 03 (2003) 051, arXiv:hep-th/0211216.

[8] R. Dijkgraaf, A. Sinkovics, and M. Temurhan, "Matrix Models and Gravitational Corrections," Adv. Theor. Math. Phys. 7 (2004) 1155-1176, arXiv:hep-th/0211241.

[9] H. Fuji and S. Mizoguchi, "Gravitational Corrections for Supersymmetric Gauge Theories with Flavors via Matrix Models," Nucl. Phys. B698 (2004) 53-91, arXiv:hep-th/0405128.

[10] E. Witten, "On S-Duality in Abelian Gauge Theory," Selecta Math. 1 (1995) 383, arXiv:hep-th/9505186.

[11] G. W. Moore and E. Witten, "Integration over the $u$-plane in Donaldson Theory," Adv. Theor. Math. Phys. 1 (1998) 298-387, arXiv:hep-th/9709193.

[12] M. Aganagic, A. Klemm, M. Mariño, and C. Vafa, "The Topological Vertex," Commun. Math. Phys. 254 (2005) 425-478, arXiv:hep-th/0305132.

[13] N. Nekrasov and A. Okounkov, "Seiberg-Witten Theory and Random Partitions," arXiv:hep-th/0306238. 
[14] R. Dijkgraaf, L. Hollands, P. Sułkowski, and C. Vafa, "Supersymmetric Gauge Theories, Intersecting Branes and Free Fermions," JHEP 02 (2008) 106, arXiv:0709.4446 [hep-th].

[15] L. F. Alday, D. Gaiotto, and Y. Tachikawa, "Liouville Correlation Functions from Four-Dimensional Gauge Theories," Lett. Math. Phys. 91 (2010) 167-197, arXiv:0906.3219 [hep-th].

[16] N. Wyllard, " $A_{N-1}$ Conformal Toda Field Theory Correlation Functions from Conformal $\mathcal{N}=2 S U(N)$ Quiver Gauge Theories," JHEP 11 (2009) 002, arXiv:0907.2189 [hep-th].

[17] G. Bonelli and A. Tanzini, "Hitchin Systems, $\mathcal{N}=2$ Gauge Theories and W-Gravity," arXiv:0909.4031 [hep-th].

[18] H. Itoyama, K. Maruyoshi, and T. Oota, "Notes on the Quiver Matrix Model and 2D-4D Conformal Connection," arXiv:0911.4244 [hep-th].

[19] L. Hollands, C. A. Keller, and J. Song, "From SO/Sp Instantons to W-Algebra Blocks," JHEP 03 (2011) 053, arXiv:1012.4468 [hep-th].

[20] L. Hollands, C. A. Keller, and J. Song, "Towards a 4d/2d correspondence for Sicilian quivers," JHEP 10 (2011) 100, arXiv:1107.0973 [hep-th].

[21] D. Gaiotto, "Asymptotically Free $\mathcal{N}=2$ Theories and Irregular Conformal Blocks," arXiv:0908.0307 [hep-th].

[22] A. Marshakov, A. Mironov, and A. Morozov, "On Non-Conformal Limit of the AGT Relations," Phys. Lett. B682 (2009) 125-129, arXiv:0909.2052 [hep-th].

[23] M. Taki, "On AGT Conjecture for Pure Super Yang-Mills and W- algebra," JHEP 05 (2011) 038, arXiv:0912.4789 [hep-th].

[24] E. B. Vinberg and V. L. Popov, "On a class of quasihomogeneous affine varieties," Math. USSR-Izv. 6 (1972) 743.

[25] quoted in Chap. III in D. Garfinkle, "A new construction of the Joseph ideal," 1982. http://hdl.handle.net/1721.1/15620

[26] S. Benvenuti, A. Hanany, and N. Mekareeya, "The Hilbert Series of the One Instanton Moduli Space," JHEP 06 (2010) 100, arXiv:1005.3026 [hep-th].

[27] M. Bershadsky and H. Ooguri, "Hidden $S L(N)$ Symmetry in Conformal Field Theories," Commun. Math. Phys. 126 (1989) 49.

[28] B. Feigin and E. Frenkel, "Quantization of the Drinfeld-Sokolov Reduction," Phys. Lett. B246 (1990) 75-81.

[29] P. Bouwknegt and K. Schoutens, "W symmetry in conformal field theory," Phys. Rept. 223 (1993) 183-276, arXiv:hep-th/9210010.

[30] K. Thielemans, "A Mathematica Package for Computing Operator Product Expansions," Int. J. Mod. Phys. C2 (1991) 787-798.

[31] E. J. Martinec and N. P. Warner, "Integrable Systems and Supersymmetric Gauge Theory," Nucl. Phys. B459 (1996) 97-112, arXiv:hep-th/9509161.

[32] A. Klemm, W. Lerche, S. Yankielowicz, and S. Theisen, "Simple singularities and N=2 supersymmetric Yang-Mills theory," Phys.Lett. B344 (1995) 169-175, arXiv:hep-th/9411048 [hep-th]. 
[33] A. Gorsky, I. Krichever, A. Marshakov, A. Mironov, and A. Morozov, "Integrability and Seiberg-Witten Exact Solution," Phys. Lett. B355 (1995) 466-474, arXiv:hep-th/9505035.

[34] H. Itoyama and A. Morozov, "Integrability and Seiberg-Witten Theory: Curves and Periods," Nucl. Phys. B477 (1996) 855-877, arXiv:hep-th/9511126.

[35] W. Lerche and N. P. Warner, "Exceptional SW Geometry from ALE Fibrations," Phys. Lett. B423 (1998) 79-86, arXiv:hep-th/9608183.

[36] T. J. Hollowood, "Strong Coupling $\mathcal{N}=2$ Gauge Theory with Arbitrary Gauge Group," Adv. Theor. Math. Phys. 2 (1998) 335-355, arXiv:hep-th/9710073.

[37] D.-E. Diaconescu, R. Donagi, and T. Pantev, "Intermediate Jacobians and ADE Hitchin Systems," arXiv:hep-th/0607159.

[38] P. Slodowy, Simple Singularities and Simple Algebraic Groups, vol. 815 of Lecture Notes in Mathematics. Springer, 1980.

[39] J. Fuchs, B. Schellekens, and C. Schweigert, "From Dynkin Diagram Symmetries to Fixed Point Structures," Commun. Math. Phys. 180 (1996) 39-98, arXiv:hep-th/9506135.

[40] N. J. Evans, C. V. Johnson, and A. D. Shapere, "Orientifolds, Branes, and Duality of 4D Gauge Theories," Nucl. Phys. B505 (1997) 251-271, arXiv:hep-th/9703210.

[41] K. Landsteiner, E. Lopez, and D. A. Lowe, " $\mathcal{N}=2$ Supersymmetric Gauge Theories, Branes and Orientifolds," Nucl. Phys. B507 (1997) 197-226, arXiv:hep-th/9705199.

[42] A. Brandhuber, J. Sonnenschein, S. Theisen, and S. Yankielowicz, "M-theory and Seiberg-Witten Curves: Orthogonal and Symplectic Groups," Nucl. Phys. B504 (1997) 175-188, arXiv: hep-th/9705232.

[43] A. Braverman, "Instanton Counting via Affine Lie Algebras I: Equivariant J-Functions of (Affine) Flag Manifolds and Whittaker Vectors," in Workshop on algebraic structures and moduli spaces: CRM Workshop, J. Hurturbise and E. Markman, eds. AMS, July, 2003. arXiv:math/0401409.

[44] A. Braverman and P. Etingof, "Instanton Counting via Affine Lie Algebras. II: from Whittaker Vectors to the Seiberg-Witten Prepotential," in Studies in Lie Theory: dedicated to A. Joseph on his 60th birthday, J. Bernstein, V. Hinich, and A. Melnikov, eds. Birkhäuser, 2006. arXiv:math/0409441.

[45] N. A. Nekrasov, "Seiberg-Witten Prepotential from Instanton Counting," Adv. Theor. Math. Phys. 7 (2004) 831-864, arXiv:hep-th/0206161.

[46] D. Finnell and P. Pouliot, "Instanton Calculations Versus Exact Results in FourDimensional SUSY Gauge Theories," Nucl. Phys. B453 (1995) 225-239, arXiv:hep-th/9503115.

[47] K. Ito and N. Sasakura, "One-Instanton Calculations in $\mathcal{N}=2$ Supersymmetric $S U\left(N_{C}\right)$ Yang-Mills Theory," Phys. Lett. B382 (1996) 95-103, arXiv:hep-th/9602073.

[48] N. Dorey, T. J. Hollowood, V. V. Khoze, and M. P. Mattis, "The Calculus of Many Instantons," Phys. Rept. 371 (2002) 231-459, arXiv:hep-th/0206063.

[49] N. Nekrasov and S. Shadchin, "ABCD of Instantons," Commun. Math. Phys. 252 (2004) 359-391, arXiv:hep-th/0404225. 
[50] M. Mariño and N. Wyllard, "A Note on Instanton Counting for $\mathcal{N}=2$ Gauge Theories with Classical Gauge Groups," JHEP 05 (2004) 021, arXiv:hep-th/0404125.

[51] C. W. Bernard, N. H. Christ, A. H. Guth, and E. J. Weinberg, "Pseudoparticle Parameters for Arbitrary Gauge Groups," Phys. Rev. D16 (1977) 2967.

[52] A. I. Vainshtein, V. I. Zakharov, V. A. Novikov, and M. A. Shifman, "ABC of Instantons," Sov. Phys. Usp. 25 (1982) 195.

[53] P. B. Kronheimer, "Instantons and the geometry of the nilpotent variety," J. Diff. Geom. 32 (1990) 473.

[54] R. Brylinski, "Instantons and Kähler geometry of nilpotent orbits," in Representation theories and algebraic geometry, vol. 514 of NATO Adv. Sci. Inst. Ser. C Math. Phys. Sci., pp. 85-125. Kluwer, 1998. math.SG/9811032.

[55] P. Kobak and A. Swann, "The hyperkähler geometry associated to Wolf spaces," Boll. Unione Mat. Ital. Serie 8, Sez. B Artic. Ric. Mat. 4 (2001) 587, math.DG/0001025.

[56] K. Ito and N. Sasakura, "Exact and Microscopic One-Instanton Calculations in $\mathcal{N}=2$ Supersymmetric Yang-Mills Theories," Nucl. Phys. B484 (1997) 141-166, arXiv:hep-th/9608054.

[57] V. G. Drinfeld and V. V. Sokolov, "Lie algebras and equations of Korteweg-de Vries type," J. Sov. Math. 30 (1984) 1975-2036.

[58] V. A. Fateev and S. L. Lukyanov, "The Models of Two-Dimensional Conformal Quantum Field Theory with $\mathbb{Z}_{N}$ Symmetry," Int. J. Mod. Phys. A3 (1988) 507.

[59] S. L. Lukyanov and V. A. Fateev, "Conformally invariant models of two-dimensional QFT with $\mathbb{Z}_{N}$ symmetry," Sov. Phys. JETP 67 (1988) 447.

[60] S. L. Lukyanov and V. A. Fateev, "Exactly solvable models of conformal quantum theory associated with the simple Lie algebra $D_{N}$," Sov. J. Nucl. Phys. 49 (1989) 925-932. [Yad.Fiz.49:1491-1504,1989].

[61] S. L. Lukyanov and V. A. Fateev, "Physics reviews: Additional symmetries and exactly soluble models in two-dimensional conformal field theory,". Chur, Switzerland: Harwood (1990) 117 p. (Soviet Scientific Reviews A, Physics: 15.2).

[62] K. Ito and S. Terashima, "Free Field Realization of $\mathrm{W} B C_{N}$ and $\mathrm{WG}_{2}$ Algebras," Phys. Lett. B354 (1995) 220-231, arXiv: hep-th/9503165.

[63] U. Bruzzo, F. Fucito, J. F. Morales, and A. Tanzini, "Multi-instanton calculus and equivariant cohomology," JHEP 05 (2003) 054, arXiv:hep-th/0211108

[64] H. Nakajima and K. Yoshioka, "Instanton counting on blowup. I," arXiv:math/0306198.

[65] D. Maulik and A. Okounkov, private communication.

[66] V. I. Arnol'd, "Critical points of functions on a manifold with boundary, the simple Lie groups $B_{k}, C_{k}, F_{4}$ and singularities of evolutes," Uspekhi Mat. Nauk 33 no. 5(203), (1978) 91.

[67] S. Mizoguchi, "Determinant Formula and Unitarity for the $W_{3}$ Algebra," Phys. Lett. B222 (1989) 226.

[68] V. G. Kac and M. Wakimoto, "Quantum reduction in the twisted case," arXiv:math-ph/0404049. 